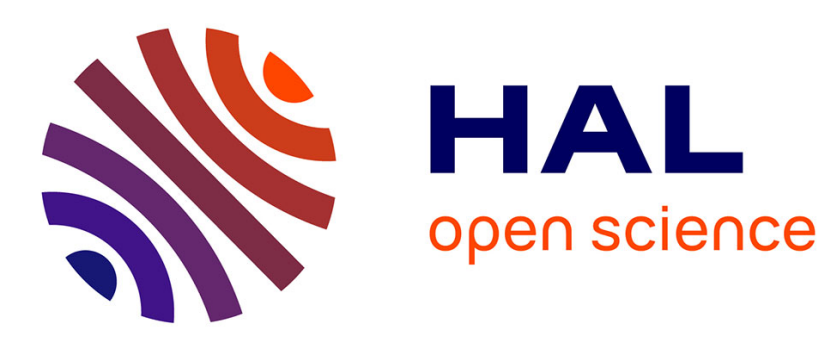

\title{
A Gurson-type criterion for porous ductile solids containing arbitrary ellipsoidal voids-II: Determination of yield criterion parameters
}

Komlanvi Madou, Jean-Baptiste Leblond

\section{- To cite this version:}

Komlanvi Madou, Jean-Baptiste Leblond. A Gurson-type criterion for porous ductile solids containing arbitrary ellipsoidal voids-II: Determination of yield criterion parameters. Journal of the Mechanics and Physics of Solids, 2012, 60 (5), pp.1037 - 1058. 10.1016/j.jmps.2012.01.010 . hal-01436394

\section{HAL Id: hal-01436394 \\ https: / hal.sorbonne-universite.fr/hal-01436394}

Submitted on 16 Jan 2017

HAL is a multi-disciplinary open access archive for the deposit and dissemination of scientific research documents, whether they are published or not. The documents may come from teaching and research institutions in France or abroad, or from public or private research centers.
L'archive ouverte pluridisciplinaire HAL, est destinée au dépôt et à la diffusion de documents scientifiques de niveau recherche, publiés ou non, émanant des établissements d'enseignement et de recherche français ou étrangers, des laboratoires publics ou privés. 


\title{
A Gurson-type criterion for porous ductile solids containing arbitrary ellipsoidal voids - II: Determination of yield criterion parameters
}

\author{
Komlanvi Madou, Jean-Baptiste Leblond * \\ UPMC Univ Paris 6 and CNRS, UMR 7190, Institut Jean Le Rond d'Alembert, F-75005 \\ Paris, France
}

\begin{abstract}
The aim of this paper is to fully determine the parameters of the approximate homogenized yield criterion for porous ductile solids containing arbitrary ellipsoidal cavities proposed in Part I. This is done through improvements of the limit-analysis of some representative hollow cell presented there. The improvements are of two kinds. For hydrostatic loadings, the limit-analysis is refined by performing micromechanical finite element computations in a number of significant cases, so as to replace Leblond and Gologanu (2008)'s trial velocity field representing the expansion of the void by the exact, numerically determined one. For deviatoric loadings, limit-analysis is dropped and direct use is made of some general rigorous results for nonlinear composites derived by Ponte-Castaneda (1991), Willis (1991) and Michel and Suquet (1992) using the earlier work of Willis (1977) and the concept of "linear comparison material". This hybrid approach is thought to lead to the best possible expressions of the yield criterion parameters. The criterion proposed reduces to (variants of) classical approximate criteria proposed by Gurson (1977) and Gologanu et al. $(1993,1994,1997)$ in the specific cases of spherical or spheroidal, prolate or oblate cavities. An overview of the validation of this criterion through micromechanical finite element computations is finally presented.
\end{abstract}

Keywords: Porous ductile solids; ellipsoidal voids; homogenization; limit-analysis; finite element computations; linear comparison material

\section{Introduction}

In Part I, we derived a homogenized Gurson-like yield criterion for plastic porous solids containing general ellipsoidal voids, by performing an approximate limit-analysis of some ellipsoidal elementary cell containing a confocal ellipsoidal cavity. However the parameters appearing in the yield function were not ascribed fully explicit expressions. The aim of the present Part II is to complete the definition of the yield criterion by providing expressions of all the coefficients involved.

\footnotetext{
* Corresponding author.
} 
The treatment of Part I was based on use of some trial incompressible velocity fields recently discovered by Leblond and Gologanu (2008), satisfying conditions of homogeneous strain rate on an arbitrary family of confocal ellipsoids. It would seem natural to still use these fields to derive explicit expressions of the yield criterion coefficients. However, although Leblond and Gologanu (2008)'s fields are irreplaceable in a first step to define the approximations leading to a Gurson-like yield function and relate its coefficients to some mathematical features of the velocity fields, their use beyond this point would introduce inaccuracies in the specific case of very flat oblate spheroidal voids. These inaccuracies were already evidenced in the works of Gologanu (1997) and Gologanu et al. (1997) by comparing the predictions of Gologanu et al. (1994)'s fields (identical to those of Leblond and Gologanu (2008) in the case considered) to the results of some numerical micromechanical simulations. Some comments on this point are in order here.

Consider first the case of a positive hydrostatic loading, the response to which was described in Part I by Leblond and Gologanu (2008)'s trial velocity field $\mathbf{v}^{0}(\mathbf{r})$. The accurate numerical results provided in Figure I.2.b of Gologanu et al. (1997) clearly show that for a penny-shaped crack (completely flat oblate spheroidal void) subjected to such a loading, this velocity field notably overestimates the local plastic dissipation in the vicinity of the void. In addition, Figure I.3.b of Gologanu et al. (1997) illustrates another wrong feature of the field $\mathbf{v}^{0}(\mathbf{r})$, schematized in Figure 1 below. It is clear from equations (9) of Part I that for an oblate spheroidal void of axis $O z$, all diagonal components $D_{x x}^{0}(\Lambda)=D_{y y}^{0}(\Lambda)$, $D_{z z}^{0}(\Lambda)$ of the overall strain rate tensor defined by Leblond and Gologanu (2008)'s field $\mathbf{v}^{0}(\mathbf{r})$ are positive; this is schematized by the black arrows in Figure 1. But Gologanu et al. (1997)'s numerical calculations reproduced in their Figure I.3.b show that if a very flat oblate spheroidal void is embedded in a moderately flat cell, the $x x$ and $y y$ components of the true strain rate tensor resulting from some positive hydrostatic loading are in fact negative; this is schematized by the dotted arrows in Figure 1.

These two undesired features of Leblond and Gologanu (2008)'s field $\mathbf{v}^{0}(\mathbf{r})$ are certainly related since a significant geometric inadequacy of some trial velocity field must generate a significant error in the associated estimate of the plastic dissipation.

With regard to deviatoric loadings, it was remarked by Gologanu (1997) that for a pennyshaped crack of axis $O z$ subjected to some pure shear stress $\Sigma_{z x}$ or $\Sigma_{z y}$, Gologanu et al. (1994)'s yield criterion, based on the same trial velocity fields as Part I of the present work, predicts no effect of the crack, that is, $\left|\Sigma_{z x}\right|$ or $\left|\Sigma_{z y}\right|=\sigma_{0} / \sqrt{3}$ where $\sigma_{0}$ denotes the microscopic yield stress in simple tension. This is tied to the simplistic description of the response to such a loading in the form of a homogeneous shear strain rate field. Such a result for $\Sigma_{z x}$ or $\Sigma_{z y}$ is in contradiction with intuition and the rigorous upper bound for this case established by Willis (1977), Ponte-Castaneda (1991), Willis (1991) and Michel and Suquet (1992).

In order to derive good values for the yield criterion parameters, it is therefore necessary to improve upon the limit-analysis of Part I, based on Leblond and Gologanu (2008)'s velocity fields. The improvements brought here will parallel, in the general ellipsoidal case, those brought by Gologanu (1997) and Gologanu et al. (1997) to Gologanu et al. (1993)'s and Gologanu et al. (1994)'s earlier models, in the spheroidal, prolate or oblate cases. 


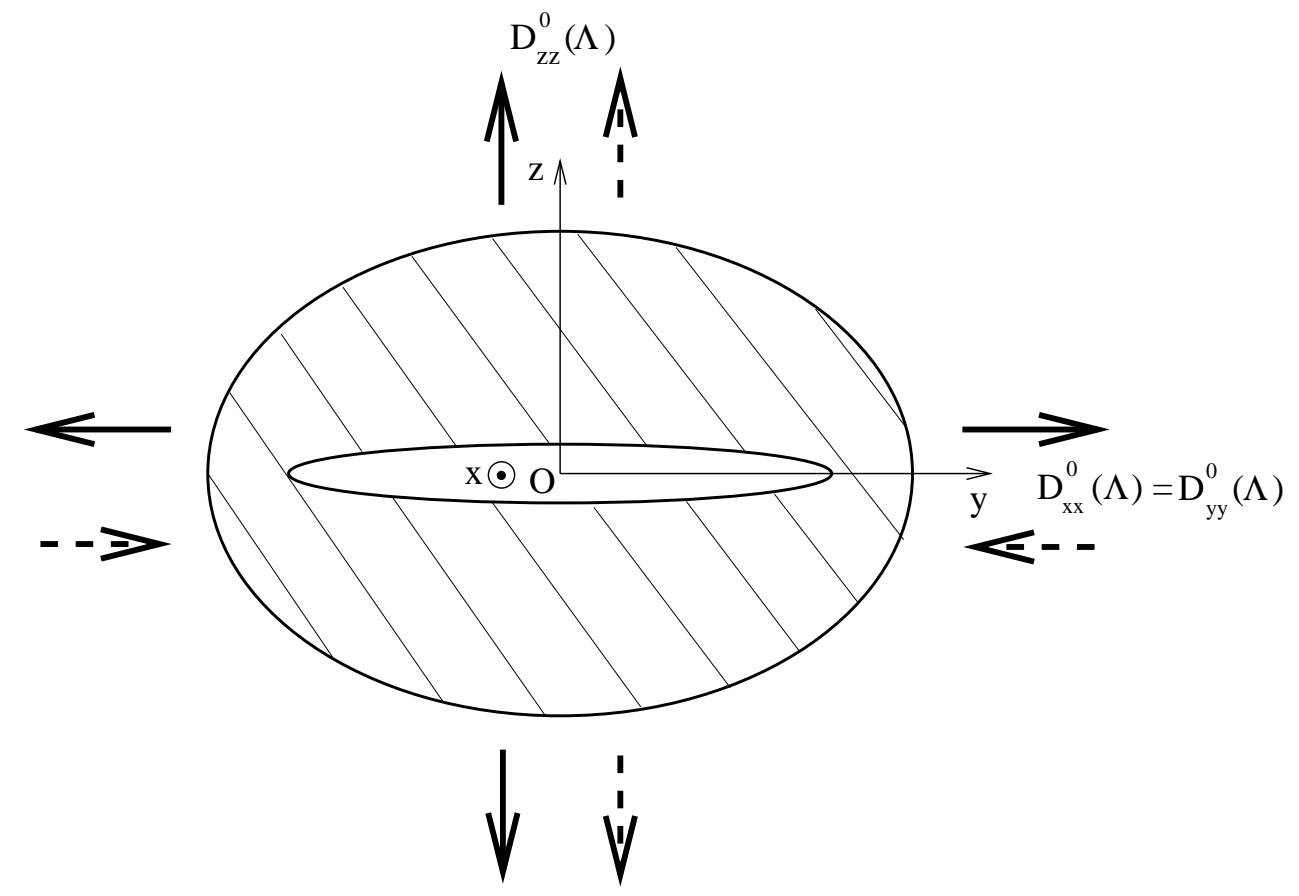

Fig. 1. A very flat oblate spheroidal void subjected to hydrostatic tension - Black arrows: components of the overall strain rate tensor in Leblond and Gologanu (2008)'s trial solution; dotted arrows: components of the true tensor

These improvements will be of two kinds. For hydrostatic loadings, the approximate limitanalysis of Part I will be refined by replacing Leblond and Gologanu (2008)'s trial velocity field $\mathbf{v}^{0}(\mathbf{r})$ by the supposedly exact field, determined numerically by the finite element method. An essential point here will be that the approximations made in Part I are not tied to the specific analytic form of Leblond and Gologanu (2008)'s field $\mathbf{v}^{0}(\mathbf{r})$ but only to some of its qualitative mathematical properties - essentially the existence and finiteness or infiniteness of some limits - which makes it possible to replace this field by another one possessing the same basic features. Computations will be performed in a number of representative cases, and heuristic but accurate analytical formulae representing the numerical results will be looked for, so as to cover all possible cases.

For deviatoric loadings, although the limit-analysis of Part I could certainly be refined in a similar way, we shall adopt a more expedient approach consisting in making direct use of Ponte-Castaneda (1991)'s, Willis (1991)'s and Michel and Suquet (1992)'s general and rigorous bound for nonlinear composites, derived from the earlier work of Willis (1977) and the concept of "linear comparison material". A number of coefficients of the yield function proposed will thus simply be fixed so as to exactly match this bound, without any reference to any specific trial velocity field.

The paper is organized as follows:

- As a preliminary, Section 2 explains how to choose the value of the constant $\chi$ of Part I to get the most geometrically natural possible definition of the "second porosity" $g$.

- Section 3 then explains the method used to perform numerical limit-analysis through finite element computations. 
- Section 4 presents the application of this method to the determination of the coefficient $\kappa$ appearing in the hyperbolic cosine of the yield function proposed.

- Section 5 then explains the determination of the coefficients $H_{x}, H_{y}, H_{z}$ appearing in the same term, by a similar method.

- Next, Section 6 expounds the application of Willis (1977)'s, Ponte-Castaneda (1991)'s, Willis (1991)'s and Michel and Suquet (1992)'s results to the determination of the quadratic form of the stress components appearing in the yield function proposed.

- The definition of this yield function being complete at this stage, Section 7 provides a summary of all relevant equations for ease of reference.

- Section 8 then specializes the criterion to various special situations. It is shown to reproduce a number of exact, or approximate but accurate results pertaining to these cases.

- Finally Section 9 presents a quick survey of the numerical validation of the yield function proposed through micromechanical finite element simulations, postponing a full exposition of the topic to some future paper.

\section{Definition of the second porosity}

The first task is to choose the value of the dimensionless constant $\chi$ appearing in the expression of the second porosity $g$ (equation (86) of Part I) so as to get the simplest possible definition of this quantity. As will be seen, this can be done simply, without appealing to numerical simulations.

Since $\chi$ appears in the expression of $g$ through its ratio to the limit $L$, a natural idea is to connect its value to that of $L$. One difficulty, however, is that as explained in Subsection 6.2 of Part I, $\chi$ must always remain comparable to unity, whereas as mentioned in Subsections 5.3 and $5.5, L$ goes to infinity in some cases. To solve this difficulty, consider the estimate $L^{\mathrm{LG}}$ of $L$ deriving from Leblond and Gologanu (2008)'s trial field $\mathbf{v}^{0}(\mathbf{r})$, provided by equation (47) of Part I ; extracting the factor $\frac{1}{k^{4}}$ from the braces and noting that $\bar{a}^{6} k^{4}=$ $\bar{a}^{2} \bar{b}^{4}$ by equation (17), one may rewrite this estimate as

$$
L^{\mathrm{LG}}=\frac{2}{\sqrt{3} \bar{a} \bar{b}^{2}}\left\{\frac{1}{k^{\prime 4}}\left[\left(k^{4}-k^{2}+1\right) E^{\prime 2}+k^{4} K^{\prime 2}-k^{2}\left(k^{2}+1\right) E^{\prime} K^{\prime}\right]+k^{2}+1\right\}^{1 / 2} .
$$

A study of the quantity $\bar{a} \bar{b}^{2} L^{\mathrm{LG}}=\frac{2}{\sqrt{3}}\{\ldots\}^{1 / 2}$ shows that it varies moderately with the parameter $k$, from $\sqrt{\frac{8}{3}} \simeq 1.63$ for $k=0$ (prolate spheroidal case) to $\sqrt{\frac{3 \pi^{2}+32}{12}} \simeq 2.27$ for $k=1$ (oblate spheroidal case). Thus suggests that some multiple of $\bar{a} \bar{b}^{2} L$ may be a suitable choice for the value of $\chi$.

More specifically, we shall adopt the value

$$
\chi \equiv \frac{3}{2} \bar{a} \bar{b}^{2} L
$$

$L$ denoting the true, numerically determined value of the limit considered. With the estimate $L^{\mathrm{LG}}$ of $L$ given by equation (1), the quantity $\chi$ thus defined varies between 
$\sqrt{6} \simeq 2.45$ for $k=0$ and $\frac{\sqrt{3\left(3 \pi^{2}+32\right)}}{4} \simeq 3.40$ for $k=1$; and numerical calculations expounded below reveal that for $k=1$, the true value of $L$ is lower ${ }^{1}$ and $\chi$ much closer to unity. Therefore equation (2) respects the requested condition that $\chi$ never be much smaller or larger than unity.

With the choice defined by equation (2), equation (86) of Part I for $g$ simply becomes

$$
g \equiv \frac{\bar{a} \bar{b}^{2}}{\Omega}
$$

Now, up to a factor of $\frac{4 \pi}{3}, \bar{a} \bar{b}^{2}$ is the volume of an ellipsoid of semi-axes $\bar{a}, \bar{b}, \bar{b}$, whereas $\Omega$ is the volume of the cell considered. Equation (3) therefore means that the second porosity $g$ is that defined by some fictitious prolate spheroidal void obtained by rotating the completely flat ellipsoid (of semi-axes $\bar{a}, \bar{b}, \bar{c}=0$ ) about its major axis. Note that this definition stands as a natural extension to general ellipsoidal voids of Budiansky and O'Connell (1976)'s "crack density parameter" applicable to penny-shaped cracks; it does however seem to be new in the general case.

In addition, with the choice (2), the expression of the variable $w$ defined by equation (68) of Part I becomes

$$
w \equiv \frac{2}{3\left(v+\bar{a} \bar{b}^{2}\right)} .
$$

Note that both equations (3) and (4) are independent of the numerical value of the limit $L$.

\section{Finite-element-based numerical limit-analysis}

Special finite element methods disregarding elasticity have been developed for numerical limit-analysis: see e.g. Pastor (1978); de Buhan and Maghous (1995); Pastor et al. (2009). In this work, however, we use the customary finite element method including elasticity available in standard commercial codes.

The most natural approach in this context would be to simulate the gradual loading of the structure up to its limit-load by a step-by-step method. Instead of doing so, however, we impose a single, large load step - without any update of the geometry since limit-analysis disregards geometric changes. The use of a single large step offers the advantage of quicker calculations (assuming, of course, that convergence of the elastoplastic iterations can be achieved for large load increments), without introducing any error of principle; indeed it is shown in Appendix A that provided that an implicit (backward Euler) algorithm is used for the projection of the elastic stress predictor onto the yield locus, the equations of the time-discretized finite element problem are exactly equivalent to those of limit-analysis.

$\overline{1}$ This is because $L$ is tied to the plastic dissipation, and Leblond and Gologanu (2008)'s trial field $\mathbf{v}^{0}(\mathbf{r})$ overestimates this dissipation for an oblate void loaded hydrostatically; see the Introduction. 
In addition, a value of Poisson's ratio very close to $1 / 2$ is used. The choice of such a value rather than a more realistic one of 0.25 or 0.3 is possible since limit-loads are known to be independent of elastic coefficients, and facilitates convergence of the elastoplastic iterations for the following reasons:

- the material being then (almost) elastically incompressible, the first elastoplastic iteration assuming a purely elastic behavior yields a displacement field already satisfying the requested incompressibility condition;

- as again explained in Appendix A, provided that the cell is entirely plastic when its limit-load is reached (which is the case in almost all calculations reported here), one may then use some not-too-large load step ensuring only that the limit-load is reached, but not necessarily that the elastic strain is negligible as compared to the plastic strain.

The structures considered are ellipsoidal cells containing a confocal ellipsoidal void and subjected to conditions of homogeneous boundary strain. Attention is restricted to overall strain tensors having principal directions identical to those of the ellipsoidal cell, implying existence of symmetries about three perpendicular planes which permit to mesh only $1 / 8$ of the structure. Use is made of the SYSTUS ${ }^{\circledR}$ finite element code developed by ESI Group, which allows the user to write higher level programmes managing and exploiting calculations. Three such programmes have been developed.

- The first prepares the mesh. Starting from a hollow sphere meshed with standard means, it displaces nodes so as to deform the geometry into an ellipsoid containing a confocal void. Nodes are placed on successive confocal ellipsoids for reasons explained in Subsection 4.1 below.

- The second programme runs the calculations. Using some Newton method, it adjusts the principal components of the overall strain tensor imposed so as match the desired values of the ratios of the overall stress components.

- Finally the third programme exploits the results obtained; among other things, it calculates the overall stress components through volume integration of the local stress components.

The initial mesh (1/8 of a hollow sphere) consists of 26,481 nodes and 24,000 trilinear 8 -node brick elements; there are 20 elements in the radial direction. The values of the material constants are as follows: Young's modulus, 210, $000 \mathrm{MPa}$; Poisson's ratio, 0.499; yield stress in simple tension, $300 \mathrm{MPa}$. The values of the macroscopic strain components imposed vary from roughly 0.1 to 1 . Good convergence of the elastoplastic iterations is obtained with typically a few hundreds of BFGS iterations.

\section{Expression of the constant $\kappa$}

\subsection{Generalities}

In Part I, the constant $\kappa$ appearing in the hyperbolic cosine of the yield function proposed was related by equation $(87)_{2}$ to some "average value" (yet requiring a precise definition) 
$\bar{F}$ of the function $F(w)$, and the estimate $F^{\mathrm{LG}}(w)$ of this function deriving from Leblond and Gologanu (2008)'s trial field $\mathbf{v}^{0}(\mathbf{r})$ was provided by equation $(69)_{1}$. With the definition (4) above of the variable $w$, the expression of this estimate becomes

$$
F^{\mathrm{LG}}(w) \equiv \frac{3}{2}\left(v+\bar{a} \bar{b}^{2}\right) \sqrt{\left\langle d_{e q}^{0}{ }^{2}(\mathbf{r})\right\rangle_{\mathcal{E}_{\lambda}}}=\frac{3}{2} \frac{v+\bar{a} \bar{b}^{2}}{\Omega} \frac{\sqrt{\left\langle d_{e q}^{\left.0{ }^{2}(\mathbf{r})\right\rangle_{\mathcal{E}_{\lambda}}}\right.}}{\operatorname{tr} \mathbf{D}^{0}(\Lambda)}
$$

where equation (10) of Part I has been used; the advantage of the final expression is that the field $\mathbf{v}^{0}(\mathbf{r})$ appears only through the ratio $\sqrt{\left\langle d_{e q}^{0}{ }^{2}(\mathbf{r})\right\rangle_{\mathcal{E}_{\lambda}}} / \operatorname{tr} \mathbf{D}^{0}(\Lambda)$ independent of the "normalization factor" adopted in its definition.

Finite element calculations are to provide, up to some multiplicative constant, the true value of the velocity field for a hydrostatic loading. From there will follow the true value of the ratio just mentioned in the form $\sqrt{\left\langle\epsilon_{e q}^{1}{ }^{2}(\mathbf{r})\right\rangle_{\mathcal{E}_{\lambda}}} / \operatorname{tr} \mathbf{E}^{1}$ where $\epsilon_{e q}^{1}(\mathbf{r})$ and $\mathbf{E}^{1}$ denote the final local equivalent strain and overall strain tensor. However, instead of defining the true function $F(w)$ as

$$
F(w) \equiv \frac{3}{2} \frac{v+\bar{a} \bar{b}^{2}}{\Omega} \frac{\sqrt{\left\langle\epsilon_{e q}^{12}(\mathbf{r})\right\rangle_{\mathcal{E}_{\lambda}}}}{\operatorname{tr} \mathbf{E}^{1}},
$$

as would seem logical, we shall define it as

$$
F(w) \equiv \frac{3}{2} \frac{v+\bar{a} \bar{b}^{2}}{\Omega} \frac{\left\langle\epsilon_{e q}^{1}(\mathbf{r})\right\rangle_{\mathcal{E}_{\lambda}}}{\operatorname{tr} \mathbf{E}^{1}}
$$

replacing thus $\sqrt{\left\langle\epsilon_{e q}^{1}{ }^{2}(\mathbf{r})\right\rangle_{\mathcal{E}_{\lambda}}}$ by $\left\langle\epsilon_{e q}^{1}(\mathbf{r})\right\rangle_{\mathcal{E}_{\lambda}}$. It may seem that this introduces an extra approximation degrading the accuracy of the limit-analysis, but in fact the converse is true. Indeed in Part I the basic definition (23) of the estimate of the overall plastic dissipation involved the average of the equivalent strain rate, not its square, and the expression (27) involving this square was arrived at only as the result of approximation $\mathcal{A}_{1}$ necessary to derive a Gurson-like estimate of the overall yield function. Thus replacing $\sqrt{\left\langle\epsilon_{e q}^{1}{ }^{2}(\mathbf{r})\right\rangle_{\mathcal{E}_{\lambda}}}$ by $\left\langle\epsilon_{e q}^{1}(\mathbf{r})\right\rangle_{\mathcal{E}_{\lambda}}$ in fact means coming back ${ }^{2}$ to the original expression of the overall plastic dissipation, thereby reducing the error introduced by approximation $\mathcal{A}_{1}$.

In practice, the arrangement of nodes over successive confocal ellipsoids will make it easy to calculate the average value $\left\langle\epsilon_{e q}^{1}(\mathbf{r})\right\rangle_{\mathcal{E}_{\lambda}}$ for various values of $\lambda$, without using its complex definition involving ellipsoidal coordinates (equation (26) of Part I). Indeed, since the local weight appearing in this definition is just the infinitesimal volume element between two nearby ellipsoids, it will suffice to take the numerical integral of $\epsilon_{e q}^{1}(\mathbf{r})$ over the elements lying between two successive ellipsoids, and then divide this integral by the total volume of these elements.

The argument of $F(w)$ is of dimension (length) ${ }^{-3}$; the search for an approximate expression of this function will be made easier by rather considering it as a function of some dimensionless variable. We thus set

$$
F(w) \equiv \tilde{F}(\tilde{w}) \quad, \quad \tilde{w} \equiv \frac{3}{2} \bar{a}^{3} w=\frac{\bar{a}^{3}}{v+\bar{a} \bar{b}^{2}}=\frac{\bar{a}^{3}}{v+k^{2} \bar{a}^{3}} .
$$

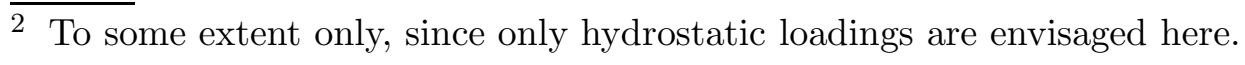


The minimum and maximum values of $\tilde{w}$ are 0 , corresponding to $v=+\infty$, and $1 / k^{2}$ $(=+\infty$ in the prolate spheroidal case), corresponding to $v=0$. Also, $F(w)$ will be plotted versus the eccentricity

$$
\epsilon_{x z}(\lambda) \equiv \epsilon_{x z} \equiv \frac{\bar{a}}{\sqrt{a^{2}+\lambda}}
$$

of the ellipsoidal surface $\mathcal{E}_{\lambda}$ in the $O x z$ plane, or some power of this parameter, rather than versus $w$ or $\tilde{w}$. The variable $\epsilon_{x z}$ is convenient for graphical purposes since its extremal values of 0 and 1 are finite and independent of $k$.

In addition to its argument $w$ or $\tilde{w}$, the function $F(w)$ theoretically depends on three geometric parameters: for instance $k$, which specifies the family of confocal ellipsoids $\mathcal{E}_{\lambda}$, and the eccentricities

$$
e_{x z} \equiv \epsilon_{x z}(\lambda=0)=\frac{\bar{a}}{a} \quad ; \quad E_{x z} \equiv \epsilon_{x z}(\lambda=\Lambda)=\frac{\bar{a}}{A}
$$

of the inner and outer ellipsoids $\mathcal{E}_{0}, \mathcal{E}_{\Lambda}$ in the $O x z$ plane, which specify these ellipsoids within the family considered; or $k, e_{x z}$ and the porosity $f$. In actual problems of ductile rupture, however, the porosity is always small, that is, the inner and outer ellipsoids are far apart. Hence one may consider the sole limiting case of a small inner ellipsoid and a large outer one, that is of a large $e_{x z}$ and a small $E_{x z}$; the function $F(w)$ then depends on the sole geometric parameter $k$.

\subsection{Prolate spheroidal case}

Figure 2 shows the function $F(w) \equiv F^{\text {prol }}(w)$ obtained numerically in the prolate spheroidal case $(k=0)$, for a void with semi-axes in the proportions 5:1:1 and a porosity of 0.005 , corresponding to inner and outer eccentricities $e_{x z} \simeq 0.98$ and $E_{x z} \simeq 0.45$ (the outer spheroid is almost spherical in spite of its moderate eccentricity). ${ }^{3}$ The figure also compares these results to the approximate analytical formula

$$
F^{\mathrm{prol}}(w) \equiv \tilde{F}^{\mathrm{prol}}(\tilde{w}) \equiv 1-\left(1-\frac{\sqrt{3}}{2}\right) \frac{\tilde{w}}{\tilde{w}+11 / 5}
$$

and the earlier one proposed by Gologanu (1997) and Gologanu et al. (1997). Equation (10) provides a slightly better representation of the numerical results and is adopted henceforth. Note that the expression proposed goes to the limits 1 and $\sqrt{3} / 2$ when the surface $\mathcal{E}_{\lambda}$ becomes a large sphere $(\tilde{w} \rightarrow 0)$ and a small cylinder $(\tilde{w} \rightarrow+\infty)$ respectively, as appropriate in such cases (see equations (34) and (58) of Part I and (5) of the present paper, noting that the approximate function $F^{\mathrm{LG}}(w)$ becomes exact in these cases).

3 These numerical results are not compared to those of Gologanu (1997) and Gologanu et al. (1997), obtained by another method. The comparison would make little sense because of a systematic error made by these authors when evaluating $\left\langle d_{e q}^{0}{ }^{2}(\mathbf{r})\right\rangle_{\mathcal{E}_{\lambda}}$, which arose from the approximate replacement of this average value by the value of $d_{e q}^{0} 2(\mathbf{r})$ at a specific point. 


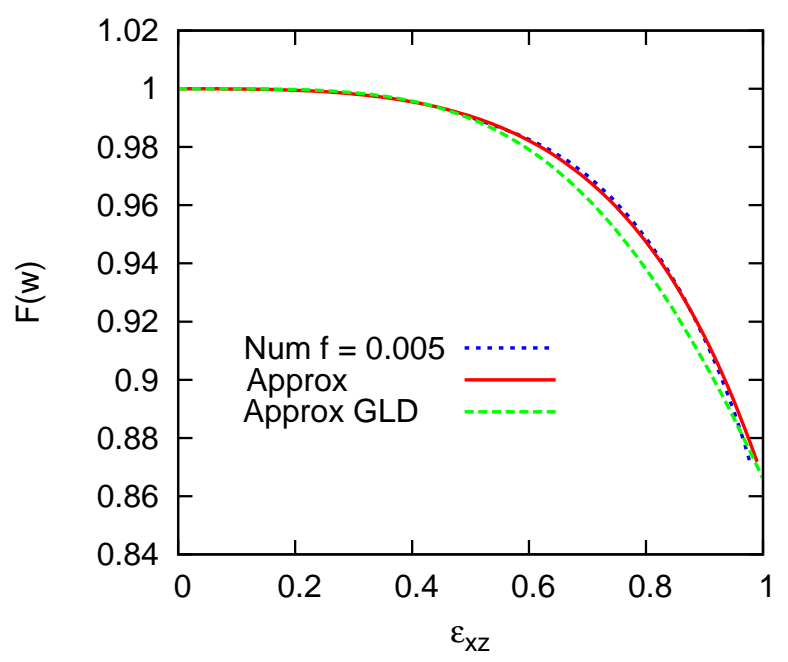

Fig. 2. The function $F(w)$ in the prolate spheroidal case - Numerical results (Num) and approximations proposed here (Approx) and in Gologanu et al. (1997)'s work (Approx GLD)

Figure 3 shows numerical results for the same void but three (small) values of the porosity. The effect of this parameter can be observed to be minor, which confirms that $F^{\text {prol }}(w)$ may be approximately considered as a function of the sole argument $w$ or $\tilde{w}$.

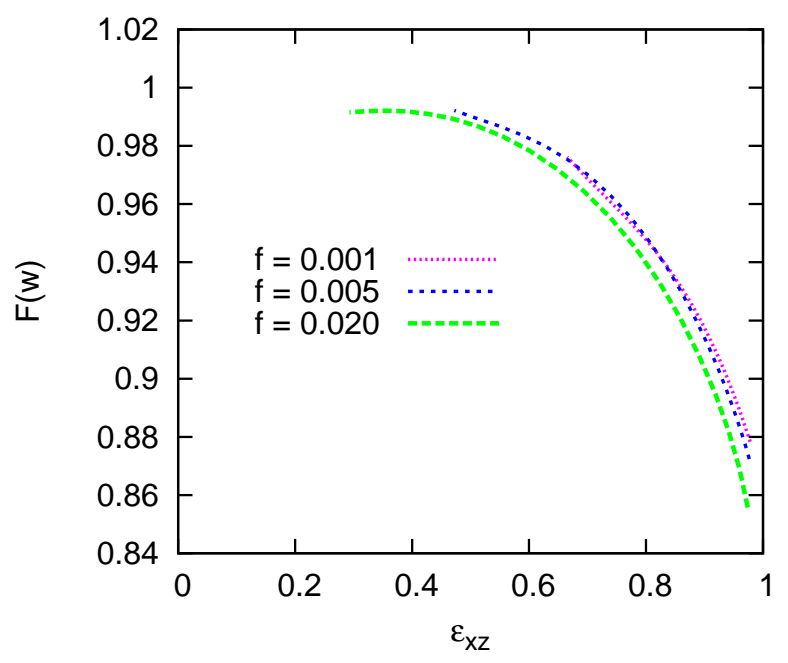

Fig. 3. The function $F(w)$ in the prolate spheroidal case - Numerical results - Influence of the porosity

\subsection{Oblate spheroidal case}

Figure 4 shows the function $F(w) \equiv F^{\mathrm{obl}}(w)$ obtained numerically in the oblate spheroidal case $(k=1)$, for a void with semi-axes in the proportions $5: 5: \frac{1}{5}$ (eccentricity $e_{x z} \simeq 0.999$ ), 
and various porosities. ${ }^{4}$ (In this figure and the next ones, the plot is versus $\epsilon_{x z}^{4}$ instead of $\epsilon_{x z}$ for a better representation for high values of $\epsilon_{x z}$ ). The influence of the porosity is larger than in the prolate case, but can still be neglected in a first approximation. The figure also compares these results to the approximate formula (adopted hereafter)

$$
F^{\mathrm{obl}}(w) \equiv \tilde{F}^{\mathrm{obl}}(\tilde{w}) \equiv 1+\frac{13}{10} \tilde{w}-\frac{3}{2} \tilde{w}^{5},
$$

and to Gologanu (1997)'s and Gologanu et al. (1997)'s earlier, less accurate one.

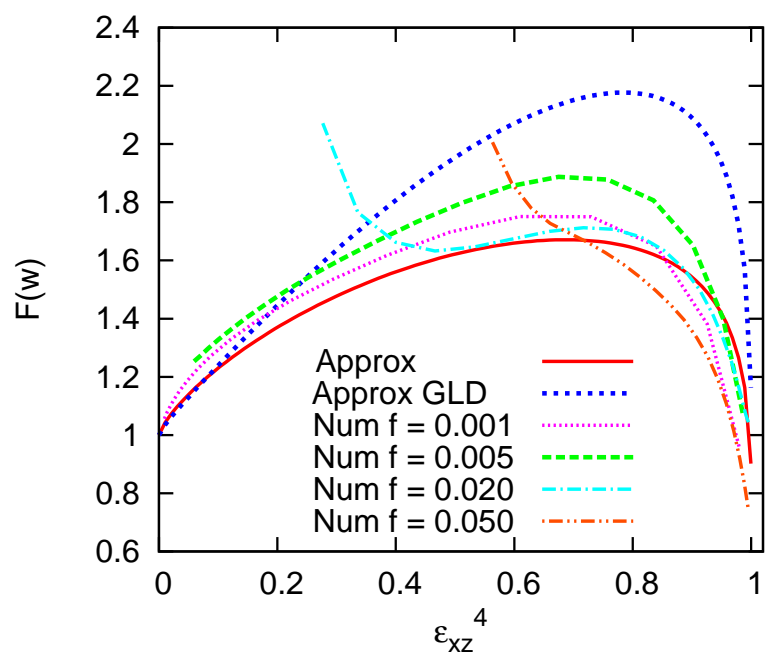

Fig. 4. The function $F(w)$ in the oblate spheroidal case - Numerical results (Num) and approximations proposed here (Approx) and in Gologanu et al. (1997)'s work (Approx GLD)

Incidentally, it is easy to show that the constant $\chi$ of equation (2) coincides with the value of $F(w)$ for $\epsilon_{x z}=1$, which Figure 4 shows to be close to unity for $k=1$, as was anticipated in Section 2.

\subsection{General case}

The voids considered in the prolate and oblate spheroidal cases had semi-axes in the

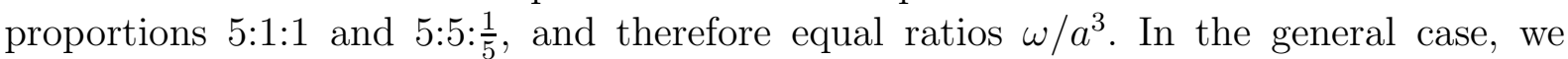
consider voids having the same value of $\omega / a^{3}$ but other values of $k$. Figure 5 shows the numerical results thus obtained for a porosity of 0.005 .

It can be observed that for values of $\epsilon_{x z}$ close to unity, the function $F(w)$ varies quickly for some values of $k$ and in a complex way; for some pairs $\left(k, \epsilon_{x z}\right)$, the value of $F(w)$ even

$\overline{4}$ These numerical results are not compared to those of Gologanu (1997) and Gologanu et al. (1997) for the same reason as before.

5 For porosities of 0.02 and 0.05 , the results shown seem to suggest that $F^{\mathrm{obl}}(w)$ diverges in the limit $\epsilon_{x z} \rightarrow 0$; this impression is erroneous just because the domain of definition of $\epsilon_{x z}$ depends on $f$ and does not exceed that shown in the figure. 


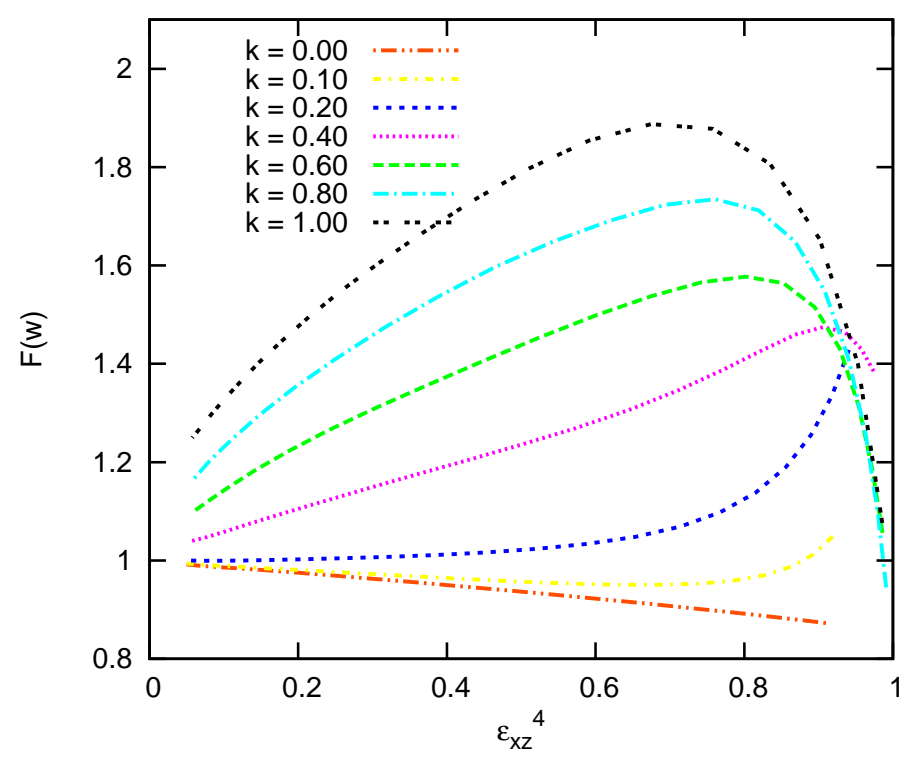

Fig. 5. The function $F(w)$ in the general case - Numerical results for a porosity of 0.005 and various values of $k$

lies outside of the interval defined by the values of $F^{\mathrm{prol}}(w)$ and $F^{\mathrm{obl}}(w)$ for the same value of $\epsilon_{x z}$. Some explanatory comments are in order here.

Consider the value of $F(w)$ in the two limit-processes $\left(\tilde{w} \rightarrow 1 / k^{2}\right.$, then $\left.k \rightarrow 0\right)$ and $(k \rightarrow 0$, then $\tilde{w} \rightarrow+\infty)$. In the first one, one considers the plastic dissipation under hydrostatic loading in the vicinity of the completely flat ellipsoid of semi axes $\bar{a}, \bar{b}, \bar{c}=0$ $\left(\tilde{w} \rightarrow 1 / k^{2}\right)$, then lets the larger dimension $\bar{a}$ of this ellipsoid go to infinity $(k \rightarrow 0)$; that is, one envisages the dissipation near a planar empty band. In the second, one lets the completely flat ellipsoid become a needle $(k \rightarrow 0)$, then considers the dissipation in the vicinity of this needle $(\tilde{w} \rightarrow+\infty)$; that is, one envisages the dissipation near a circular cylindrical void. Clearly, the two situations are completely distinct; therefore one must expect that

$$
\lim _{k \rightarrow 0} \lim _{\tilde{w} \rightarrow 1 / k^{2}} \tilde{F}(k ; \tilde{w}) \neq \lim _{\tilde{w} \rightarrow+\infty} \lim _{k \rightarrow 0} \tilde{F}(k ; \tilde{w})
$$

where explicit indications of dependence upon $k$ have temporarily been introduced. The non-coincidence of these limits is confirmed by consideration of the estimate $F^{\mathrm{LG}}(w)$ of the function $F(w)$ resulting from Leblond and Gologanu (2008)'s trial field $\mathbf{v}^{0}(\mathbf{r})$, for which they are easily checked to amount to $\sqrt{6}$ and $\sqrt{3} / 2$ respectively.

Now values of the parameter $\tilde{w}$ close to $1 / k^{2}$ are equivalent to values of the eccentricity $\epsilon_{x z}$ close to unity. Therefore equation (12) means that the limits $k \rightarrow 0$ and $\epsilon_{x z} \rightarrow 1$ do not commute for the function $F(w)$. This implies existence of a boundary layer in the graph of this function for values of $\epsilon_{x z}$ close to unity, within which it varies quickly; this boundary layer becomes infinitely thin in the limit $k \rightarrow 0$. This explains the complexity of the behavior of $F(w)$ noted above.

The existence of this boundary layer makes it impossible to look for an approximate analytical expression of $F(w)$ in the form of a simple interpolation between those of 
$F^{\mathrm{prol}}(w)$ and $F^{\mathrm{obl}}(w)$. However Figure 6 shows that the more complex formula

$$
F(w) \equiv \tilde{F}(\tilde{w}) \equiv(1-k) \tilde{F}^{\text {prol }}(\tilde{w})+k \tilde{F}^{\mathrm{obl}}\left(k^{2} \tilde{w}\right)+k^{2}(1-k)^{2} \frac{3 \tilde{w}}{8-5 k^{2} \tilde{w}}
$$

with $\tilde{F}^{\text {prol }}$ and $\tilde{F}^{\text {obl }}$ given by equations (10) and (11), provides an acceptable approximate representation of the numerical results for several representative values of $k$. The expression proposed consists of two parts. The first is a kind of interpolation between $F^{\text {prol }}(w)$ and $F^{\mathrm{obl}}(w)$; the factor $k^{2}$ in $\tilde{F}^{\mathrm{obl}}\left(k^{2} \tilde{w}\right)$ ensures that the argument of $\tilde{F}^{\text {obl }}$ is between 0 and 1 , as it must. The second part is a homographic function of $\tilde{w}$, the role of which is to approximately account for the boundary layer. This function is zero for $\tilde{w}=0$ (so as to respect the condition $\tilde{F}(0)=1$ ) and for $k=0$ or 1 (as is necessary for $\tilde{F}(\tilde{w}$ ) to reduce to $\tilde{F}^{\operatorname{prol}}(\tilde{w})$ and $\tilde{F}^{\mathrm{obl}}(\tilde{w})$ in these cases), but it is important when $\tilde{w}$ is close to $1 / k^{2}$ and $k$ differs from zero and unity. Observe that equation (13) does respect condition (12), the values of the left- and right-hand sides being $\sqrt{3} / 2+1$ and $\sqrt{3} / 2$ respectively. ${ }^{6}$

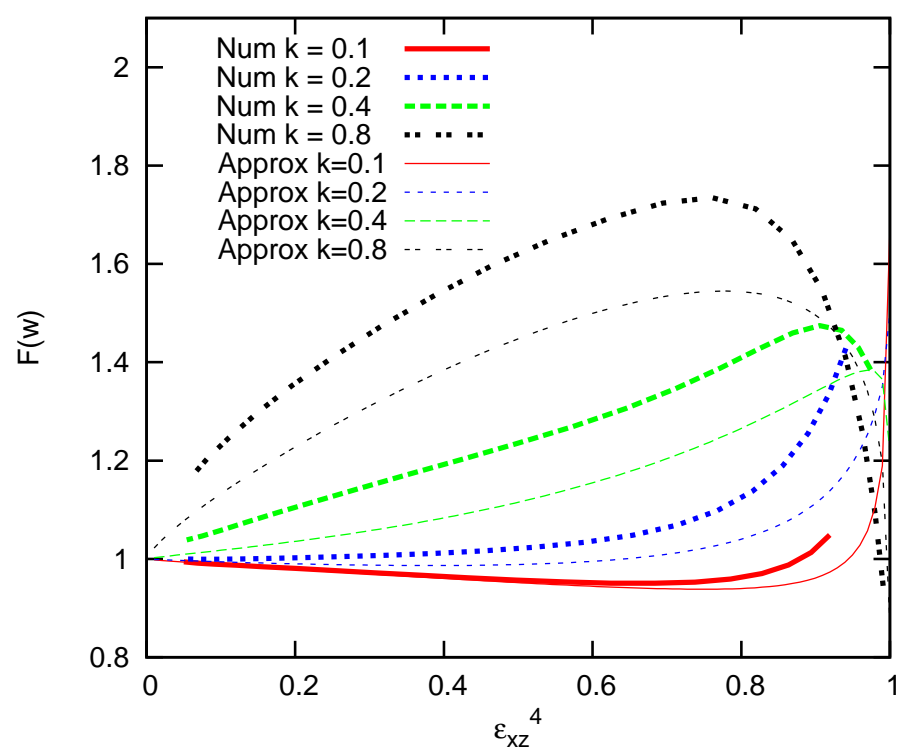

Fig. 6. The function $F(w)$ in the general case - Numerical results (Num) for a porosity of 0.005 and approximation proposed (Approx)

Figure 7 illustrates, for the typical value $k=0.6$, the dependence of the numerical results upon the porosity. Comparing Figures 6 and 7, one sees that the intrinsic error made when adopting the approximate formula (13) for $F(w)$ is comparable to that resulting from neglect of the influence of the porosity upon this function.

$\overline{6}$ The first value differs somewhat from that, $\sqrt{6}$, corresponding to the estimate $F^{\mathrm{LG}}(w)$ of the function $F(w)$, see above; requiring the coincidence would be pointless since Leblond and Gologanu (2008)'s field $\mathbf{v}^{0}(\mathbf{r})$ is not expected to represent the exact solution for a planar empty band loaded hydrostatically. 


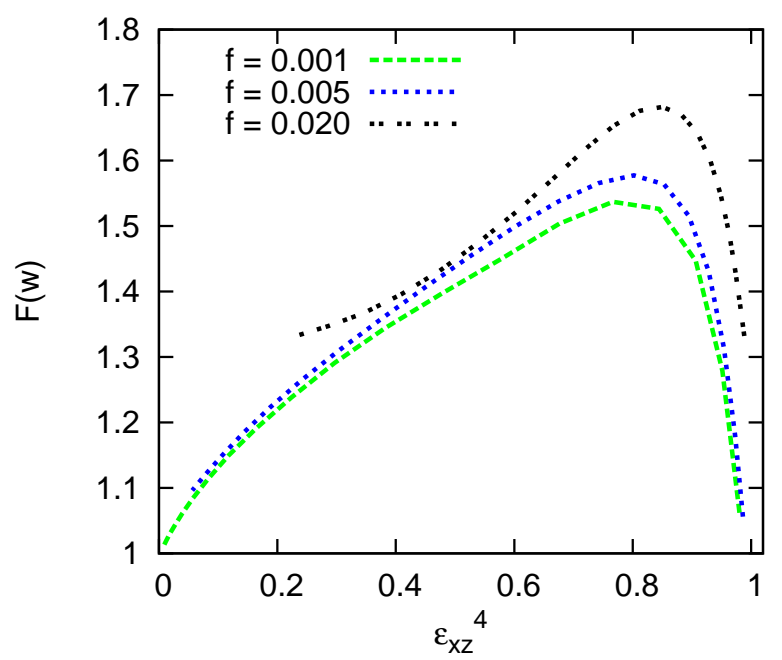

Fig. 7. The function $F(w)$ for $k=0.6$ - Numerical results - Influence of the porosity

\subsection{Approximate formula for $\kappa$}

The function $F(w)$ being represented by the approximate formula (13), one must now ascribe a precise definition to its "average value" $\bar{F}$. We follow Gologanu (1997) and Gologanu et al. (1997) here.

To be optimal, the definition of $\bar{F}$ must minimize the error resulting from approximation $\mathcal{A}_{3}$ of Part I, that is from the simplification of the expression (81) of $\Pi^{++}(\mathbf{D})$ obtained through replacement of $F(w)$ by $\bar{F}$ in the expression (70) of $\left\langle d_{e q}^{2}(\mathbf{r})\right\rangle_{\mathcal{E}_{\lambda}}$. It is clear that such a minimization cannot be achieved for all values of the kinematic parameters $\mathcal{A}, \mathcal{B}$, $\mathcal{C}, \Delta_{x y}, \Delta_{y z}, \Delta_{z x}$ simultaneously; but in the most interesting case where $\mathcal{A}$ is the sole nonzero parameter, the error can be made zero by requesting that $\bar{F}$ satisfy the condition

$$
\int_{w_{\min }}^{w_{\max }} F(w) \frac{d w}{w}=\int_{w_{\min }}^{w_{\max }} \bar{F} \frac{d w}{w}
$$

or equivalently, using the variable $\tilde{w}$ instead of $w$,

$$
\bar{F} \equiv \frac{1}{\ln \left(\tilde{w}_{\max } / \tilde{w}_{\min }\right)} \int_{\tilde{w}_{\min }}^{\tilde{w}_{\max }} \tilde{F}(\tilde{w}) \frac{d \tilde{w}}{\tilde{w}}
$$

Using equations $(3),(4)$ and $(7)_{2}$ to express $\tilde{w}_{\min }$ and $\tilde{w}_{\max }$ as

$$
\tilde{w}_{\min }=\frac{g}{k^{2}(1+g)} \quad ; \quad \tilde{w}_{\max }=\frac{g}{k^{2}(f+g)},
$$

then formulae (10), (11) and (13) to calculate the integral in equation (14), one gets

$$
\begin{aligned}
\bar{F}=1+\frac{1}{\ln \left(g_{f} / g_{1}\right)} & {\left[-(1-k)\left(1-\frac{\sqrt{3}}{2}\right) \ln \frac{11 k^{2}+5 g_{f}}{11 k^{2}+5 g_{1}}\right.} \\
& \left.+\frac{3}{5}(1-k)^{2} \ln \frac{8-5 g_{1}}{8-5 g_{f}}+\frac{13}{10} k\left(g_{f}-g_{1}\right)-\frac{3}{10} k\left(g_{f}^{5}-g_{1}^{5}\right)\right]
\end{aligned}
$$


where

$$
g_{1} \equiv \frac{g}{1+g} \quad ; \quad g_{f} \equiv \frac{g}{f+g} .
$$

Equation (15) raises a problem for a prolate spheroidal void; indeed for such a void, $g=0$ by equation (3) and $g_{1}=g_{f}=0$ by equations (16), so that the fraction $\frac{11 k^{2}+5 g_{f}}{11 k^{2}+5 g_{1}}$ is of the form $\frac{0}{0}$. The indeterminate form may be evaluated by taking $k \neq 0$, expressing $g_{1}$ and $g_{f}$ in equation (15) in terms of $k, f$ and the void eccentricity $e_{x z}$, and taking the limit $k \rightarrow 0$; one thus gets

$$
\bar{F}=1+\frac{1-\sqrt{3} / 2}{\ln f} \ln \frac{11+5 e_{x z}^{3} /\left(1-e_{x z}^{2}\right)}{11+5 f e_{x z}^{3} /\left(1-e_{x z}^{2}\right)} \quad \text { (prolate spheroidal void) }
$$

Once $\bar{F}$ is known, the constant $\kappa$ readily follows from equation $(87)_{2}$ of Part I.

\section{Expressions of the constants $H_{x}, H_{y}, H_{z}$}

\subsection{Generalities}

The estimates of the constants $H_{x}, H_{y}, H_{z}$ of the approximate yield function proposed deriving from Leblond and Gologanu (2008)'s trial field $\mathbf{v}^{0}(\mathbf{r})$ were provided by equation $(87)_{4}$ of Part I, where $\Omega=1 / \operatorname{tr} \mathbf{D}^{0}(\Lambda)$ by equation (10). The equivalent formulae for the exact values of these constants are obviously

$$
H_{x}=\frac{E_{x x}^{1}}{\operatorname{tr} \mathbf{E}^{1}} \quad ; H_{y}=\frac{E_{y y}^{1}}{\operatorname{tr} \mathbf{E}^{1}} \quad ; H_{z}=\frac{E_{z z}^{1}}{\operatorname{tr} \mathbf{E}^{1}}
$$

where $\mathbf{E}^{1}$ again denotes the final overall strain tensor determined by the finite element method for a hydrostatic loading. The value of this tensor is directly connected to the homogeneous strain conditions imposed on the cell boundary.

Just like the function $F(w)$, the constants $H_{x}, H_{y}, H_{z}$ depend on three geometric parameters, for instance $k, e_{x z}$ and $E_{x z}$, or $k, E_{x z}$ and $f$. However equation (18) makes it clear that they are determined by the restriction of the velocity field over the outer ellipsoid $\mathcal{E}_{\Lambda}$. Since the inner ellipsoid $\mathcal{E}_{0}$ is much smaller, they may be anticipated to depend little on its precise location and size, and will therefore be looked for as functions of the sole parameters $k$ and $E_{x z}$ characterizing the shape of the outer ellipsoid.

The sum of $H_{x}, H_{y}$ and $H_{z}$ being unity by equation (88) of Part I (or equation (18)), we shall concentrate on the sole determination of $H_{x}$ and $H_{y}$. 


\subsection{Prolate spheroidal case}

In the prolate spheroidal case $(k=0)$, the equivalence of the axes $O y$ and $O z$ implies that the coefficients $H_{y} \equiv H_{y}^{\text {prol }}$ and $H_{z} \equiv H_{z}^{\text {prol }}$ are equal. By equation (88) of Part I, this implies that the coefficients $H_{x} \equiv H_{x}^{\text {prol }}$ and $H_{y}^{\text {prol }}$ are connected through the relation

$$
H_{x}^{\text {prol }}=1-2 H_{y}^{\text {prol }}
$$

so that it suffices to concentrate on the second coefficient.

Figure 8 shows the numerical results obtained for $H_{y}^{\text {prol }}$ for a porosity of 0.02 , together with those obtained by Gologanu (1997) and Gologanu et al. (1997) for a porosity of 0.01 by a different method. The similarity of results illustrates their reliability. The figure also compares these results to the approximate analytical formula (adopted henceforth)

$$
H_{y}^{\mathrm{prol}} \equiv \frac{1}{3}\left(1+E_{x z}^{2}-\frac{E_{x z}^{4}}{2}\right)
$$

and that proposed by Gologanu (1997) and Gologanu et al. (1997). Observe that expression (20) goes to the correct limits $1 / 3$ and $1 / 2$ when the cell becomes a sphere $\left(E_{x z} \rightarrow 0\right)$ and a circular cylinder $\left(E_{x z} \rightarrow 1\right)$, respectively. (The value of $1 / 3$ for a hollow sphere arises from equivalence of the axes $O x, O y$ and $O z$ in this case, and that of $1 / 2$ for a hollow circular cylinder from the well-known exact solution for such a structure, loaded hydrostatically).

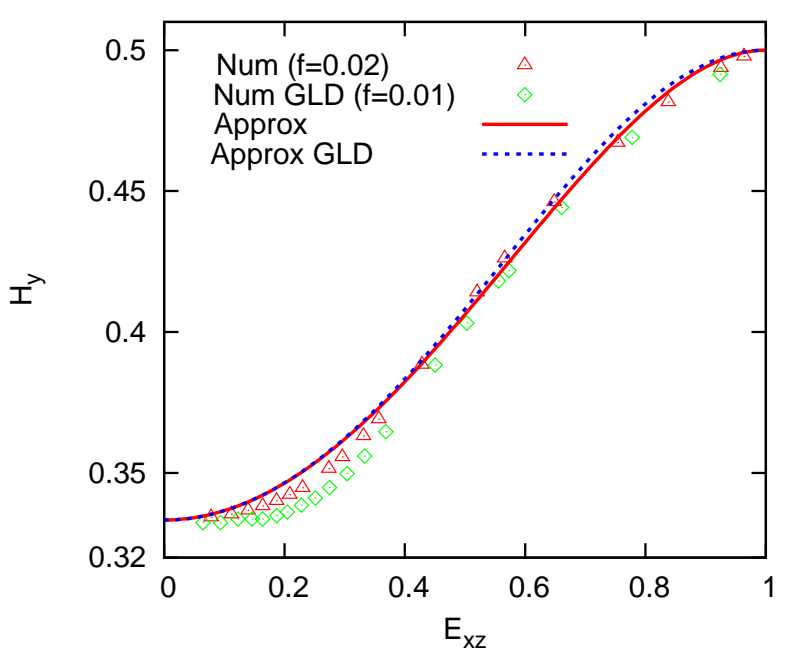

Fig. 8. The coefficient $H_{y}$ in the prolate spheroidal case - Numerical results and approximation proposed in this work (Num, Approx) and Gologanu et al. (1997)'s earlier one (Num GLD, Approx GLD)

Gologanu (1997) and Gologanu et al. (1997) also studied the influence of the porosity upon $H_{y}^{\text {prol }}$ and found it to be quite minor, which confirms that this coefficient approximately depends on the sole parameter $E_{x z}$. 


\subsection{Oblate spheroidal case}

In the oblate spheroidal case $(k=1)$, the axes $O x$ and $O y$ are equivalent so that

$$
H_{x} \equiv H_{x}^{\mathrm{obl}}=H_{y} \equiv H_{y}^{\mathrm{obl}}
$$

hence it again suffices to concentrate on the coefficient $H_{y}^{\text {obl }}$.

Figure displays the numerical results obtained for $H_{y}^{\text {obl }}$ in this work for a porosity of 0.02 and the earlier one of Gologanu (1997) and Gologanu et al. (1997) for a porosity of 0.01, and compares them to the approximate formula (adopted henceforward)

$$
H_{y}^{\mathrm{obl}} \equiv \frac{1}{3} \frac{2-7 E_{x z}^{2}+5 E_{x z}^{4}}{2-7 E_{x z}^{2}+10 E_{x z}^{4}}
$$

and the earlier one of Gologanu (1997) and Gologanu et al. (1997). Again, these is little difference between the old and new numerical results. On the other hand the new approximate formula (22) gives decidedly better results than the old one, especially for high values of $E_{x z}$, although the latter already correctly captured the change of sign of $H_{y} \cdot{ }^{7}$ Again, note that expression (22) goes to the correct limits $1 / 3$ and 0 when the cell becomes a sphere $\left(E_{x z} \rightarrow 0\right)$ and a "sandwich" consisting of two infinite planar layers of material surrounding an empty one $\left(E_{x z} \rightarrow 1\right)$, respectively. (The value of 0 for a sandwich arises from the fact that the deformation mode of such a structure, loaded hydrostatically, is a simple extension in the direction $z$ perpendicular to the layers).

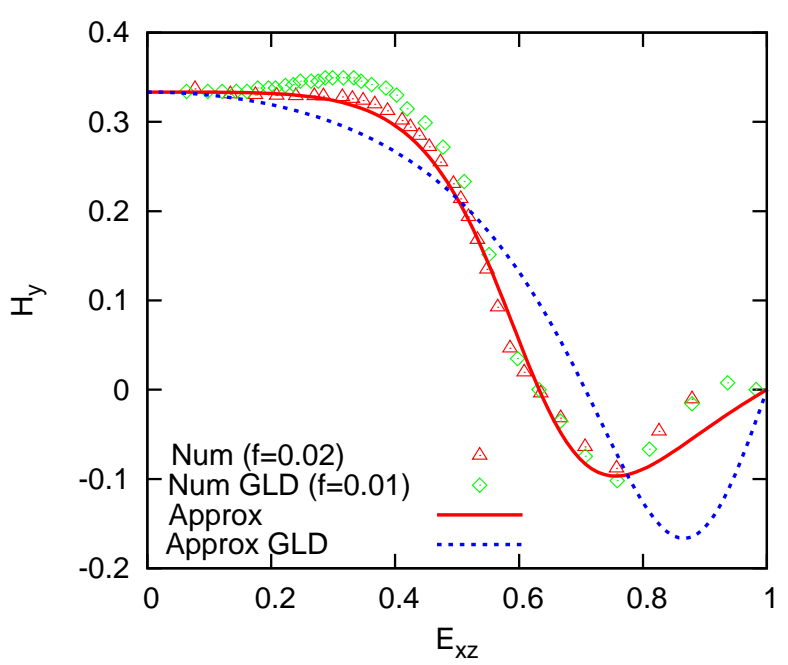

Fig. 9. The coefficient $H_{y}$ in the oblate spheroidal case - Numerical results and approximation proposed in this work (Num, Approx) and Gologanu et al. (1997)'s earlier one (Num GLD, Approx GLD)

Formula (22) is somewhat more complex than (20); correct reproduction of the numerical results in Figure 9 with a polynomial of low order would be impossible because of the

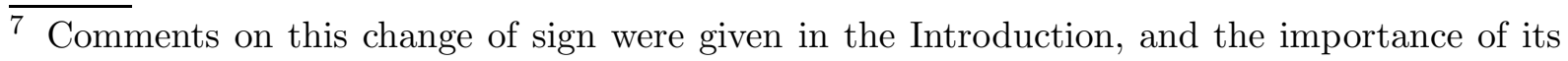
correct reproduction in a constitutive model was emphasized. 
flatness of the curves in the region of low $E_{x z}$.

Again, the influence of the porosity upon the coefficient $H_{y}^{\text {obl }}$ was studied by Gologanu (1997) and Gologanu et al. (1997), who found it to be quite minor.

\subsection{General case}

In the general case where $k$ is neither zero nor unity, the coefficients $H_{x}$ and $H_{y}$ are independent and must be studied separately.

Figure 11 shows the numerical results obtained for various values of $k$ and a porosity of 0.02. The behavior of the coefficient $H_{x}$ as a function of $k$ and $E_{x z}$ is relatively simple but that of the other coefficient is not: for $k=0.6$ for instance, when $E_{x z}$ increases, $H_{y}$ first increases, then decreases, then increases again; some curves cross each other twice; and for some pairs $\left(k, E_{x z}\right)$, the value of $H_{y}$ does not lie between those of $H_{y}^{\text {prol }}$ and $H_{y}^{\text {obl }}$ for the same value of $E_{x z}$. Again, explanatory comments on this complex behavior are in order.

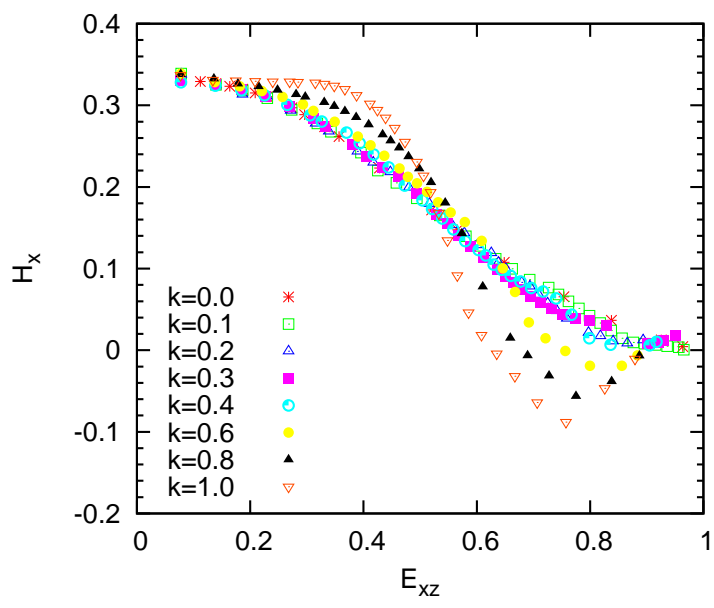

(a) The coefficient $H_{x}$

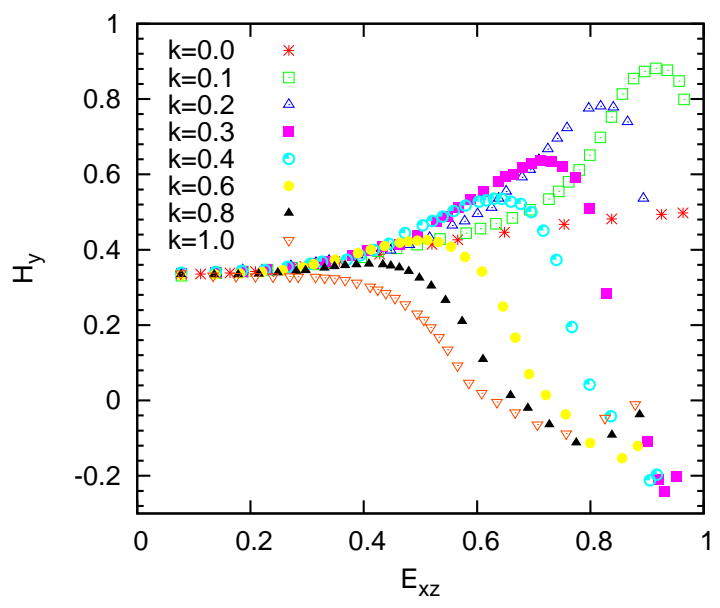

(b) The coefficient $H_{y}$

Fig. 10. The coefficients $H_{x}$ and $H_{y}$ in the general case - Numerical results for a porosity of 0.02 and various values of $k$

Assume that $k \neq 0$, thereby excluding the prolate spheroidal case, and let $E_{x z}$ go to unity. Then, whatever the value of $k$, the cell becomes a sandwich, which has been observed to undergo, under hydrostatic loading, a pure extension in the direction $z$ perpendicular to the layers; it follows that

$$
H_{x}\left(k ; E_{x z}=1\right)=H_{y}\left(k ; E_{x z}=1\right)=0 \quad(k \neq 0)
$$

where temporary indications of dependence upon $k$ and $E_{x z}$ have been introduced. On the other hand, if $k=0$, the cell is a prolate spheroid which, when $E_{x z}$ goes to unity, 
becomes a circular cylinder having

$$
H_{x}\left(k=0 ; E_{x z}=1\right) \equiv H_{x}^{\text {prol }}\left(E_{x z}=1\right)=0 ; H_{y}\left(k=0 ; E_{x z}=1\right) \equiv H_{y}^{\text {prol }}\left(E_{x z}=1\right)=\frac{1}{2} .
$$

It follows from equations (23) and (24) that

$$
\left\{\begin{array}{l}
\lim _{k \rightarrow 0} \lim _{E_{x z} \rightarrow 1} H_{x}\left(k, E_{x z}\right)=\lim _{E_{x z} \rightarrow 1} \lim _{k \rightarrow 0} H_{x}\left(k, E_{x z}\right)=0 \\
\lim _{k \rightarrow 0} \lim _{E_{x z} \rightarrow 1} H_{y}\left(k, E_{x z}\right)=0 \neq \lim _{E_{x z} \rightarrow 1} \lim _{k \rightarrow 0} H_{y}\left(k, E_{x z}\right)=\frac{1}{2} .
\end{array}\right.
$$

Like for the function $F(w)$, the non-commutation of the limits $k \rightarrow 0$ and $E_{x z} \rightarrow 1$ for the coefficient $H_{y}$ implies existence of a boundary layer in the graph representing it versus $E_{x z}$, for values of $E_{x z}$ close to unity; this layer, within which $H_{y}$ varies quickly, becomes infinitely thin when $k$ goes to zero. (In Figure 10(b), the presence of the boundary layer becomes clearer if the curves corresponding to nonzero values of $k$ are mentally supplemented with a final point $\left(E_{x z}=1, H_{y}=0\right)$, in agreement with equation $(23)_{2}$; this point could not obtained numerically because of the finiteness of the cells considered).

Figure 11(a) shows that owing to its relatively simple behavior, the coefficient $H_{x}$ may be adequately represented for various typical values of $k$ by the interpolation formula

$$
H_{x} \equiv\left(1-k^{2}\right) H_{x}^{\mathrm{prol}}+k^{2} H_{x}^{\mathrm{obl}}
$$

where the coefficients $H_{x}^{\text {prol }}$ and $H_{x}^{\text {obl }}$ are provided by equations (19), (20), (21) and (22). The coefficients $1-k^{2}$ and $k^{2}$ here allow for a better reproduction of the numerical results than simply $1-k$ and $k$.

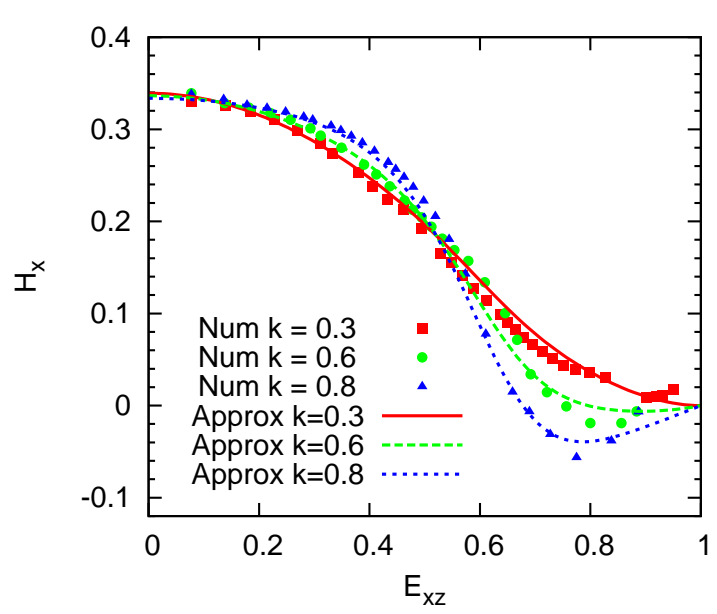

(a) The coefficient $H_{x}$

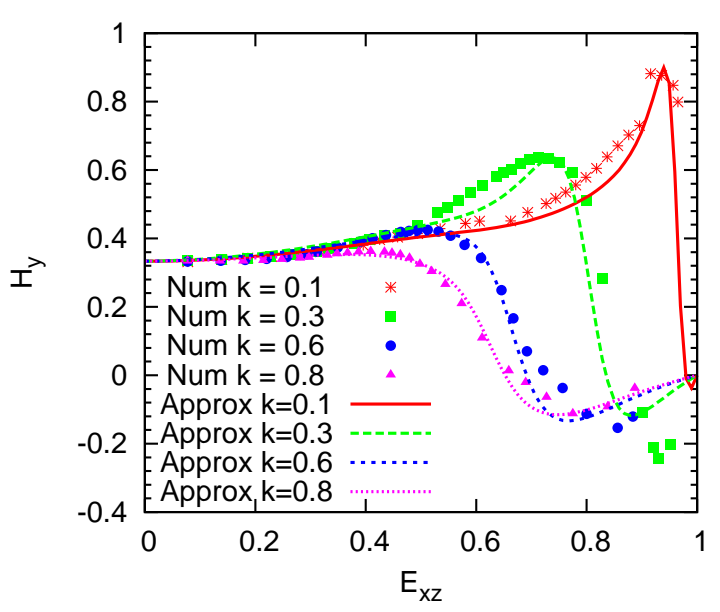

(b) The coefficient $H_{y}$

Fig. 11. The coefficient $H_{x}$ in the general case - Numerical results (Num) and approximation proposed (Approx)

The more complex behavior of the coefficient $H_{y}$ rules out such a simple representation. But Figure 11(b) shows that it may be acceptably represented for various values of $k$ by 
the formula

$$
H_{y} \equiv(1-k) H_{y}^{\mathrm{prol}}+k H_{y}^{\mathrm{obl}}+\frac{1}{2}(1-k) \frac{\alpha^{2}+\beta^{2}}{\alpha} \frac{E_{x z}^{3 / 2}\left(1-\alpha-E_{x z}\right)}{\left(1-\alpha-E_{x z}\right)^{2}+\beta^{2}}
$$

where $H_{y}^{\text {prol }}$ and $H_{y}^{\text {obl }}$ are given by equations (20) and (22), and the coefficients $\alpha$ and $\beta$ by

$$
\alpha \equiv \frac{4 k^{2}}{1+9 k^{2}} \quad ; \quad \beta \equiv \frac{3 k^{2}}{1+30 k^{2}} .
$$

Expression (27) of $H_{y}$ consists like that, (13), of $F(w)$, of an interpolation between $H_{y}^{\text {prol }}$ and $H_{y}^{\text {obl }}$ plus an extra term the role of which is to approximately account for the boundary layer. This term is zero for $E_{x z}=0$ because of the factor $E_{x z}^{3 / 2} 8$ (so as to ensure a value of $H_{y}$ of $1 / 3$ for a spherical cell) and for $k=0$ or 1 (in order for $H_{y}$ to reduce to $H_{y}^{\text {prol }}$ or $H_{y}^{\text {obl }}$ in these cases), but it is important when $E_{x z}$ is close to unity and $k$ differs from zero and unity. The factor $\frac{1}{2}(1-k) \frac{\alpha^{2}+\beta^{2}}{\alpha}$ is adjusted so as to satisfy condition $(23)_{2}$ for every $k \neq 0$. The type of dependence of coefficients $\alpha$ and $\beta$ upon $k$ ensures, among other things, that the size of the zone where $H_{y}$ varies quickly goes to zero with $k$.

Formula (27) raises a problem for an elliptic cylindrical void; indeed for such a void $k$ goes to zero (since $\bar{a}$ goes to infinity) but the cell eccentricity $E_{x z}$ simultaneously goes to unity, so that both fractions $\frac{\alpha^{2}+\beta^{2}}{\alpha}$ and $\frac{E_{x z}^{3 / 2}\left(1-\alpha-E_{x z}\right)}{\left(1-\alpha-E_{x z}\right)^{2}+\beta^{2}}$ are of the form $\frac{0}{0}$. The indeterminate forms may be evaluated by expressing $E_{x z}$ in terms of $k$ and the eccentricity

$$
E_{y z} \equiv \frac{\bar{b}}{B}
$$

of the outer ellipsoid in the $O y z$ plane, prior to taking the limit; the result reads

$$
H_{y}=\frac{1}{2}\left[1-\frac{25}{2} \frac{E_{y z}^{2}\left(9 E_{y z}^{2}-1\right)}{\left(9 E_{y z}^{2}-1\right)^{2}+36 E_{y z}^{4}}\right] \quad \text { (elliptic cylindrical void). }
$$

Finally, Figure 12 illustrates the effect of the porosity upon the coefficient $H_{y}$ for the typical value $k=0.4$. Comparison of Figures $11(\mathrm{~b})$ and 12 shows that the error resulting from use of the approximate formula (27) for this coefficient is comparable to that arising from neglect of its dependence upon the porosity.

\section{Expression of the quadratic form $\mathcal{Q}(\Sigma)$}

\subsection{Principle of the determination of $\mathcal{Q}(\boldsymbol{\Sigma})$}

In order to improve the limit-analysis of Part I for deviatoric loadings, it would certainly be possible to replace the trial uniform strain rate field used there by Eshelby (1957)'s

$\overline{8}$ The exponent $3 / 2$ here gives better results than 1 or 2 . 


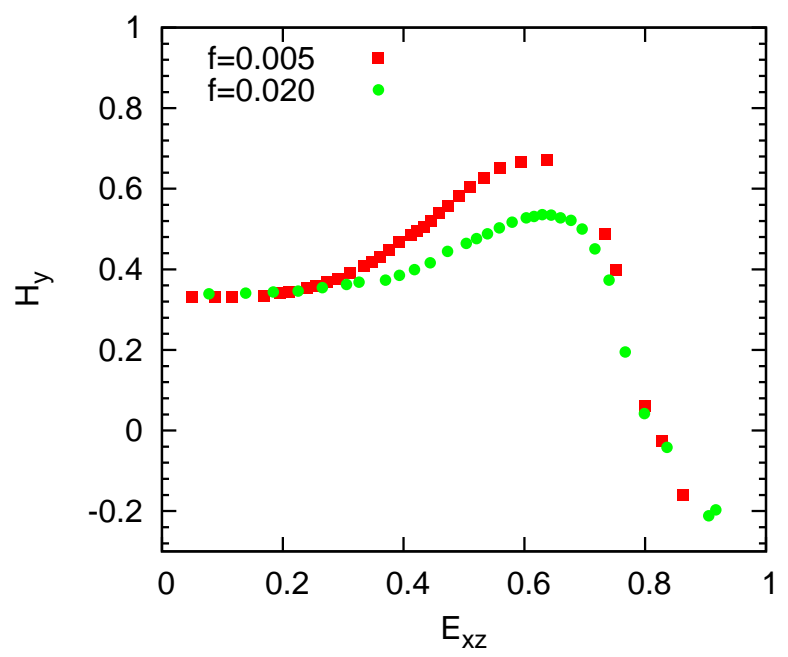

Fig. 12. The coefficient $H_{y}$ for $k=0.4$ - Numerical results - Effect of the porosity

elastic field, as was done by Monchiet et al. $(2007,2011)$ in the simpler case of spherical or spheroidal voids. We shall not however pursue such an approach and adopt instead a more expedient one consisting in dropping limit-analysis and using some general, rigorous bound for nonlinear composites, specialized to the case of plastic porous materials, as a reference. The coefficients of the quadratic form $\mathcal{Q}(\boldsymbol{\Sigma})$ appearing in the expression of the yield function proposed (equation (85) of Part I) will simply be determined so as to match this bound. No reference will be made to any specific trial velocity field.

It should be noted that the adjustment of the quadratic form $\mathcal{Q}(\boldsymbol{\Sigma})$ proposed is not the only way of retrieving one bound or another. It has indeed been shown by Monchiet et al. $(2007,2011)$ that the same result may be obtained by replacing the linear form $\mathcal{L}(\boldsymbol{\Sigma})$ in the hyperbolic cosine of the approximate yield function proposed by the square root of some quadratic form. The present choice is made because it is anticipated to allow for an easier numerical implementation of the model into some finite element programme.

Several bounds are available in the literature; the problem of choosing a suitable one will be envisaged in Subsection 6.2 below. We shall be content here to write the associated approximate yield criterion in the form

$$
\Phi^{B}(\boldsymbol{\Sigma}) \equiv \frac{\mathcal{Q}^{B}(\boldsymbol{\Sigma})}{\sigma_{0}^{2}}-(1-f)^{2}=0
$$

where $\mathcal{Q}^{B}(\boldsymbol{\Sigma})$ denotes some positive-definite quadratic form of the components of the overall stress tensor. (All available bounds are of this form, the basic reason being that they are obtained from estimates of the elastic energy of some linear comparison material, which is necessarily quadratic in the stress components). The approximate yield function $\Phi^{B}(\boldsymbol{\Sigma})$ defined by equation (31) is assumed to represent a lower estimate of the true one $\Phi(\boldsymbol{\Sigma})$, providing an exterior estimate of the yield locus $\left(\Phi^{B}(\boldsymbol{\Sigma}) \leq \Phi(\boldsymbol{\Sigma})\right.$, so that if $\Phi(\boldsymbol{\Sigma})$ is negative, so is $\Phi^{B}(\boldsymbol{\Sigma})$ ).

The bounds available in the literature are known to provide accurate predictions for 
predominantly deviatoric loadings, but not so for predominantly hydrostatic ones. Now for the latter type of loadings $\Sigma_{x x} \simeq \Sigma_{y y} \simeq \Sigma_{z z}$ so that, by equations (87) 3 and (88) of Part I, $\Sigma_{h} \simeq \Sigma_{m} \equiv \frac{1}{3} \operatorname{tr} \Sigma$; thus those loadings for which the bounds are inaccurate have a large $\left|\Sigma_{h}\right|$ as well as a large $\left|\Sigma_{m}\right|$. It is therefore logical to use the approximate yield criterion (31) as a reference for small values of $\left|\Sigma_{h}\right|$ only. More precisely, we shall enforce coincidence of the approximate yield functions $\Phi(\boldsymbol{\Sigma})$ and $\Phi^{B}(\boldsymbol{\Sigma})$ up to second order in $\Sigma_{h} / \sigma_{0}$.

Using equations (85) and (87) of Part I and expanding $\Phi(\boldsymbol{\Sigma})$ to second order, one gets

$$
\begin{aligned}
\Phi(\boldsymbol{\Sigma}) & =\frac{\mathcal{Q}(\boldsymbol{\Sigma})}{\sigma_{0}^{2}}+2(1+g)(f+g)\left(1+\frac{\kappa^{2} \Sigma_{h}^{2}}{2 \sigma_{0}^{2}}\right)-(1+g)^{2}-(f+g)^{2}+O\left[\left(\frac{\Sigma_{h}}{\sigma_{0}}\right)^{4}\right] \\
& =\frac{1}{\sigma_{0}^{2}}\left[\mathcal{Q}(\boldsymbol{\Sigma})+(1+g)(f+g) \kappa^{2} \Sigma_{h}^{2}\right]-(1-f)^{2}+O\left[\left(\frac{\Sigma_{h}}{\sigma_{0}}\right)^{4}\right] .
\end{aligned}
$$

Identifying the right-hand side (without the term $O\left[\left(\Sigma_{h} / \sigma_{0}\right)^{4}\right]$ ) to expression (31), one gets

$$
\mathcal{Q}(\boldsymbol{\Sigma}) \equiv \mathcal{Q}^{B}(\boldsymbol{\Sigma})-(1+g)(f+g) \kappa^{2} \Sigma_{h}^{2},
$$

which fully relates the coefficients of the quadratic form $\mathcal{Q}(\boldsymbol{\Sigma})$ to those of the reference one $\mathcal{Q}^{B}(\boldsymbol{\Sigma})$, in addition to the coefficients $g, \kappa, H_{x}, H_{y}, H_{z}$ already defined in Sections 2 , 4 and 5 above.

To facilitate the study of the approximate yield function thus defined, one may rewrite it in the form

$$
\begin{aligned}
\Phi(\boldsymbol{\Sigma}) & =\frac{\mathcal{Q}^{B}(\boldsymbol{\Sigma})}{\sigma_{0}^{2}}+2(1+g)(f+g)\left[\cosh \left(\frac{\kappa \Sigma_{h}}{\sigma_{0}}\right)-\frac{\kappa^{2} \Sigma_{h}^{2}}{2 \sigma_{0}^{2}}\right]-(1+g)^{2}-(f+g)^{2} \\
& =\frac{\mathcal{Q}^{B}(\boldsymbol{\Sigma})}{\sigma_{0}^{2}}-(1-f)^{2}+2(1+g)(f+g)\left[\cosh \left(\frac{\kappa \Sigma_{h}}{\sigma_{0}}\right)-1-\frac{\kappa^{2} \Sigma_{h}^{2}}{2 \sigma_{0}^{2}}\right] .
\end{aligned}
$$

A number of nice properties then become apparent:

- The yield function $\Phi(\boldsymbol{\Sigma})$ is a sum of two terms, $\mathcal{Q}^{B}(\boldsymbol{\Sigma}) / \sigma_{0}^{2}-(1-f)^{2} \equiv \Phi^{B}(\boldsymbol{\Sigma})$ and $2(1+g)(f+g)[\ldots]$, both of which are convex functions of $\boldsymbol{\Sigma}$ (the first because the quadratic form $\mathcal{Q}^{B}(\boldsymbol{\Sigma})$ is positive-definite, the second because the function $\boldsymbol{\Sigma} \mapsto \kappa \Sigma_{h} / \sigma_{0}$ is linear and the function $x \mapsto \cosh x-1-x^{2} / 2$ convex). Hence it is convex, which ensures convexity of the associated elasticity domain $\Phi(\boldsymbol{\Sigma})<0$, whatever the reference bound chosen.

- The term $2(1+g)(f+g)[\ldots]$ is non-negative, so that if $\Phi(\boldsymbol{\Sigma})$ is negative, so is $\Phi^{B}(\boldsymbol{\Sigma})$; in other words the approximate yield function $\Phi(\boldsymbol{\Sigma})$ respects the bound chosen for all possible stress states. This marks a difference with the earlier GLD model for spheroidal voids, a version of which did use some reference bound like here, but in a different way not ensuring automatic respect of this bound for all stress states (Gologanu, 1997).

- In addition, it is clear from equation (32) that if the coefficients of the quadratic form $\mathcal{Q}^{B}(\boldsymbol{\Sigma})$ are well-defined for all values of the geometric parameters, so are those of the quadratic form $\mathcal{Q}(\boldsymbol{\Sigma})$. Again, this marks a difference with the GLD yield function, the coefficients of which were noted by Monchiet (2008) to become ill-defined for very large values (of admittedly minor practical interest) of the porosity. 


\subsection{Choice of a suitable bound}

Three bounds for nonlinear composites are available in the literature:

- The classical so-called Voigt bound (see e.g. Ponte-Castaneda and Suquet (1998)). This bound relies on the sole assumption of macroscopic isotropy of the voided medium.

- The Willis bound, originally established by Willis (1977) for elastic solids and later extended by Ponte-Castaneda (1991), Willis (1991) and Michel and Suquet (1992) to nonlinear types of behavior. ${ }^{9}$ This bound applies to the case where the voids are ellipsoids of identical shape and orientation, and the distribution function of their centers possesses an "ellipsoidal symmetry" similar to that of the voids themselves. (Intuitively speaking, this means that the void spacings in the various principal directions of the voids are in the same proportions as their principal axes).

- The Ponte-Castaneda-Willis bound, established by Ponte-Castaneda and Willis (1995) in the linear case and extended by Ponte-Castaneda and Suquet (1998) to the general case. This bound stands as an extension of the Willis bound to the case where the distribution function of the centers of the voids has an arbitrary ellipsoidal symmetry independent of their shape.

Any of these bounds may be adopted to define the reference quadratic form $\mathcal{Q}^{B}(\boldsymbol{\Sigma})$ of equation (32). However the Voigt bound is a crude one, which notably wrongly predicts that circular cracks orthogonal to the direction $z$ have no effect upon the overall yield stress under pure shear loadings $\Sigma_{z x}$ or $\Sigma_{z y}$. At the other extreme the Ponte-CastanedaWillis bound is perhaps unnecessarily refined for the present case. Indeed the improvement it brings with respect to the simpler Willis bound resides in the incorporation of the influence of the spatial distribution of voids; now this distribution has even more rarely been characterized experimentally than the aspect ratios of the voids, and its effect has been argued by Danas and Ponte-Castaneda (2008) to be minor for the small porosities of practical interest. We therefore propose to adopt as a reference the Willis bound, which seems to represent a good compromise between simplicity and realism. The expression of the quadratic form $\mathcal{Q}^{W}(\boldsymbol{\Sigma})$ corresponding to this bound is provided in Appendices B and C.

It must be acknowledged that some of the hypotheses underlying the nonlinear Willis bound are somewhat inconsistent with those introduced above:

- the hypothesis of identity of the ellipsoidal symmetries of the voids themselves and the distribution function of their centers is incompatible with ours of confocality of the inner and outer ellipsoids; ${ }^{10}$

\footnotetext{
9 This bound was adopted as a reference in the model for plastic porous materials proposed by Ponte-Castaneda and Zaidman (1994); but this was done for all possible stress states, not only for predominantly deviatoric ones, resulting in large inaccuracies for predominantly hydrostatic loadings.

10 This deficiency could be remedied by adopting the Ponte-Castaneda-Willis bound instead of that of Willis as a reference, at the expense of additional complexity (notably the introduction of two Eshelby tensors instead of just one).
} 
- more basically, the nonlinear Willis bound does not rely on an intrinsically approximate analysis of some "representative cell" subjected to conditions of homogeneous boundary strain rate, but on rigorous homogenization of some unbounded medium. ${ }^{11}$

These deficiencies are thought to be of little importance because they pertain to the influence of regions located far from the voids, which again is expected to be minor for small porosities.

\section{Summary}

The definition of the yield criterion proposed being now complete, a summary of all relevant equations is provided for ease of reference. The material is rigid-ideal-plastic and obeys the von Mises yield criterion with yield stress $\sigma_{0}$ in simple tension, and the associated flow rule. The void is ellipsoidal, with semi-axes $a, b, c$ parallel to the directions $x, y, z$; the porosity is denoted $f$.

\subsection{Geometric parameters}

* Semi-axes of the outer ellipsoidal representative cell:

$$
A=\sqrt{a^{2}+\Lambda} \quad ; \quad B=\sqrt{b^{2}+\Lambda} \quad ; \quad C=\sqrt{c^{2}+\Lambda}
$$

where $\Lambda$ is the unique positive solution of the equation

$$
\left(a^{2}+\Lambda\right)\left(b^{2}+\Lambda\right)\left(c^{2}+\Lambda\right)-\frac{a^{2} b^{2} c^{2}}{f^{2}}=0
$$

* Semi-axes and shape parameter of the completely flat confocal ellipsoid:

$$
\bar{a}=\sqrt{a^{2}-c^{2}} \quad ; \quad \bar{b}=\sqrt{b^{2}-c^{2}} \quad ; \quad(\bar{c}=0) \quad ; \quad k=\frac{\bar{b}}{\bar{a}} .
$$

(Note that for an elliptic cylindrical void, $k=0$ since $\bar{a}=+\infty$ ).

* Second porosity and related quantities:

$$
g=\frac{\bar{a} \bar{b}^{2}}{A B C} \quad ; \quad g_{1}=\frac{g}{1+g} \quad ; \quad g_{f}=\frac{g}{f+g} .
$$

(Note that for an elliptic cylindrical void, $g=\frac{\bar{b}^{2}}{B C}$ since $\bar{a} \sim A$ ).

* Eccentricities of the inner and outer ellipsoids:

\footnotetext{
$\overline{11}$ The difference appears for instance when one compares the Hashin-Shtrikman bound for an infinite macroscopically isotropic voided elastic solid loaded in shear, and the exact solution for a hollow elastic sphere subjected to the same loading through conditions of homogeneous boundary strain; see Garajeu (1995).
} 


$$
e_{x z}=\frac{\bar{a}}{a} \quad ; \quad E_{x z}=\frac{\bar{a}}{A} \quad ; \quad E_{y z}=\frac{\bar{b}}{B}
$$

7.2 Expression of the yield criterion

$$
\Phi(\boldsymbol{\Sigma})=\frac{\mathcal{Q}(\boldsymbol{\Sigma})}{\sigma_{0}^{2}}+2(1+g)(f+g) \cosh \left[\frac{\mathcal{L}(\boldsymbol{\Sigma})}{\sigma_{0}}\right]-(1+g)^{2}-(f+g)^{2}=0 .
$$

\subsection{Expression of the linear form $\mathcal{L}(\boldsymbol{\Sigma})$}

* Connection to other quantities:

$$
\mathcal{L}(\boldsymbol{\Sigma})=\kappa \Sigma_{h}, \quad \kappa \equiv \frac{3}{2 \bar{F}}, \quad \Sigma_{h} \equiv H_{x} \Sigma_{x x}+H_{y} \Sigma_{y y}+H_{z} \Sigma_{z z} .
$$

* Expression of $\bar{F}$ :

$$
\begin{aligned}
\bar{F}=1+\frac{1}{\ln \left(g_{f} / g_{1}\right)}[ & -(1-k)\left(1-\frac{\sqrt{3}}{2}\right) \ln \frac{11 k^{2}+5 g_{f}}{11 k^{2}+5 g_{1}} \\
+ & \left.\frac{3}{5}(1-k)^{2} \ln \frac{8-5 g_{1}}{8-5 g_{f}}+\frac{13}{10} k\left(g_{f}-g_{1}\right)-\frac{3}{10} k\left(g_{f}^{5}-g_{1}^{5}\right)\right] .
\end{aligned}
$$

Special case: for a prolate spheroidal void,

$$
\bar{F}=1+\frac{1-\sqrt{3} / 2}{\ln f} \ln \frac{11+5 e_{x z}^{3} /\left(1-e_{x z}^{2}\right)}{11+5 f e_{x z}^{3} /\left(1-e_{x z}^{2}\right)} .
$$

* Expressions of $H_{x}, H_{y}, H_{z}$ :

$$
\left\{\begin{aligned}
H_{x}= & \left(1-k^{2}\right) H_{x}^{\mathrm{prol}}+k^{2} H_{x}^{\mathrm{obl}}, \quad H_{x}^{\mathrm{prol}}=1-2 H_{y}^{\mathrm{prol}} \quad, \quad H_{x}^{\mathrm{obl}}=H_{y}^{\mathrm{obl}} \\
H_{y}= & (1-k) H_{y}^{\mathrm{prol}}+k H_{y}^{\mathrm{obl}}+\frac{1}{2}(1-k) \frac{\alpha^{2}+\beta^{2}}{\alpha} \frac{E_{x z}^{3 / 2}\left(1-\alpha-E_{x z}\right)}{\left(1-\alpha-E_{x z}\right)^{2}+\beta^{2}} \\
& \alpha=\frac{4 k^{2}}{1+9 k^{2}} \quad, \quad \beta=\frac{3 k^{2}}{1+30 k^{2}} \\
H_{z}= & 1-H_{x}-H_{y}
\end{aligned}\right.
$$

where

$$
H_{y}^{\mathrm{prol}}=\frac{1}{3}\left(1+E_{x z}^{2}-\frac{E_{x z}^{4}}{2}\right) \quad ; \quad H_{y}^{\mathrm{obl}}=\frac{1}{3} \frac{2-7 E_{x z}^{2}+5 E_{x z}^{4}}{2-7 E_{x z}^{2}+10 E_{x z}^{4}} .
$$

(Note that for a prolate spheroidal void, $H_{y}$ reduces to $H_{y}^{\text {prol }}$ since $k$ and $\frac{\alpha^{2}+\beta^{2}}{\alpha}$ are zero).

Special case: for an elliptic cylindrical void,

$$
H_{y}=\frac{1}{2}\left[1-\frac{25}{2} \frac{E_{y z}^{2}\left(9 E_{y z}^{2}-1\right)}{\left(9 E_{y z}^{2}-1\right)^{2}+36 E_{y z}^{4}}\right] .
$$




\subsection{Expression of the quadratic form $\mathcal{Q}(\boldsymbol{\Sigma})$}

* Connection to Willis's quadratic form $\mathcal{Q}^{W}(\boldsymbol{\Sigma})$ :

$$
\mathcal{Q}(\boldsymbol{\Sigma}) \equiv \mathcal{Q}^{W}(\boldsymbol{\Sigma})-(1+g)(f+g) \kappa^{2} \Sigma_{h}^{2}
$$

* Expression of Willis's quadratic form $\mathcal{Q}^{W}(\boldsymbol{\Sigma})$ : see Appendices B and C.

\section{Special cases}

For a number of special void geometries, exact results or approximate but accurate models are available and can serve as references to which the predictions of the yield criterion proposed may be compared, using the equations just summarized. The following conclusions are reached:

- For a spherical void, the yield criterion reduces to

$$
\left(1+\frac{2 f}{3}\right) \frac{\Sigma_{e q}^{2}}{\sigma_{0}^{2}}+2 f \cosh \left(\frac{3}{2} \frac{\Sigma_{m}}{\sigma_{0}}\right)-1-f^{2}=0
$$

where $\Sigma_{e q}$ and $\Sigma_{m}$ denote the overall von Mises equivalent and mean stresses, respectively. This is exactly Leblond et al. (1994)'s slightly improved version of Gurson (1977)'s criterion for spherical voids, respecting the "nonlinear Hashin-Shtrikman bound" (Willis's nonlinear bound for a macroscopically isotropic medium).

- For a circular cylindrical void, the criterion reduces to

$\frac{\Sigma_{e q}^{2}}{\sigma_{0}^{2}}+3 f \frac{\left(\Sigma_{y y}-\Sigma_{z z}\right)^{2} / 4+\Sigma_{x y}^{2}+\Sigma_{y z}^{2}+\Sigma_{z x}^{2}}{\sigma_{0}^{2}}+2 f \cosh \left(\frac{\sqrt{3}}{2} \frac{\Sigma_{y y}+\Sigma_{z z}}{\sigma_{0}}\right)-1-f^{2}=0$.

For axisymmetric stress states $\left(\Sigma_{x x} \neq \Sigma_{y y}=\Sigma_{z z}, \Sigma_{x y}=\Sigma_{y z}=\Sigma_{z x}=0\right)$, this expression exactly reduces to Gurson (1977)'s criterion for cylindrical voids, which is known to be exact in this case. For general stress states, it respects the "nonlinear cylindrical Hashin-Shtrikman bound" (Willis's nonlinear bound for a transversely isotropic medium) provided e.g. by Leblond et al. (1994).

- For a sandwich (as defined in Subsection 5.3 above), the criterion reduces to

$$
\Sigma_{z x}=\Sigma_{z y}=\Sigma_{z z}=0 \quad ; \quad \Sigma_{e q}^{2} \equiv \Sigma_{x x}^{2}+\Sigma_{y y}^{2}-\Sigma_{x x} \Sigma_{y y}+3 \Sigma_{x y}^{2}=(1-f)^{2} \sigma_{0}^{2},
$$

which is an exact result for such a geometry.

- For a spheroidal, prolate or oblate void, it is similar to the GLD criterion (Gologanu et al., 1993, 1994; Gologanu, 1997; Gologanu et al., 1997) in general form, albeit not in detail because of different expressions of the various coefficients.

In addition, there are two void geometries for which the limit-load is known for special loading directions only, due to uniformity of the microscopic stress field in these cases:

- For an elliptic cylindrical void subjected to coaxial tension or compression (all $\Sigma_{i j}=0$ except $\Sigma_{x x}$ ), the limit-load is obviously given by 


$$
\left|\Sigma_{x x}\right|=(1-f) \sigma_{0}
$$

The criterion proposed may be checked to reproduce this exact result.

- For a circular or elliptic crack loaded in plane stress $\left(\Sigma_{z x}=\Sigma_{z y}=\Sigma_{z z}=0\right)$, the situation is more complex. Since the loadings considered automatically satisfy the boundary conditions on the crack, the criterion is then exactly that of a homogeneous plate in plane stress, that is

$$
\Sigma_{e q}^{2} \equiv \Sigma_{x x}^{2}+\Sigma_{y y}^{2}-\Sigma_{x x} \Sigma_{y y}+3 \Sigma_{x y}^{2}=\sigma_{0}^{2} .
$$

Willis's criterion $(31)$ (with $\mathcal{Q}^{B}(\boldsymbol{\Sigma}) \equiv \mathcal{Q}^{W}(\boldsymbol{\Sigma})$ ) may be checked to reproduce this result. But the criterion proposed doesn't. Indeed considering the form $(33)_{2}$ of the yield function, one sees that it predicts the same limit-load as that of Willis consisting of the sole first two terms, only when the third term $2(1+g)(f+g)[\ldots]$ is zero, that is when $\Sigma_{h}=0$; but this is not true here since $\Sigma_{h}=H_{x} \Sigma_{x x}+H_{y} \Sigma_{y y}$ where all quantities are nonzero.

The criterion proposed however reproduces the exact result (34) when the second porosity $g$ becomes either very small, because the influence of the crack disappears and the criterion reduces to that of von Mises, or very large, because the geometric configuration tends to a sandwich for which the criterion has been noted to be exact. It follows that the violation of the result (34), being zero in both limits $g \rightarrow 0$ and $g \rightarrow+\infty$, should be slight for generic values of $g$.

It may be noted that the GLD criterion was somewhat superior for (circular) cracks in that the method of determination of its parameters automatically warranted exact respect of the result (34) for all values of $g$ (Gologanu, 1997; Gologanu et al., 1997). The slight inferiority of the new model in this respect is the price to pay for the fact that its parameters have well-defined expressions for all possible values of the geometric parameters, unlike those of the GLD model (see Subsection 6.1 above).

\section{Numerical validation of the yield criterion - An overview}

The method and programmes described in Section 3 may be used to numerically determine not only the parameters of the analytical Gurson-like yield function, but also the (supposedly exact) yield locus in a number of representative cases, in order to assess the accuracy of the approximate one proposed. This has been done in eight different cases, but for space reasons only one will be envisaged here. Our purpose is merely to illustrate the kind of results obtained in a typical case, a full exposition of these results being postponed to some future paper.

We thus consider an ellipsoidal cell containing a confocal ellipsoidal void with semi-axes in the proportions 10:2:1 and a porosity of 0.01 , loaded through conditions of homogeneous boundary strain. The yield locus of this cell is a $5 \mathrm{D}$ surface in the $6 \mathrm{D}$ space of overall stress tensors, the full numerical determination of which is impossible. The problem is therefore reduced by assuming the principal axes of the overall stress tensor to coincide with those of the inner and outer ellipsoids, that is its off-diagonal components $\Sigma_{x y}, \Sigma_{y z}, \Sigma_{z x}$ to be zero. Even so, the yield locus is still a $2 \mathrm{D}$ surface in the 3D 
space of diagonal components $\left(\Sigma_{x x}, \Sigma_{y y}, \Sigma_{z z}\right)$, the full determination of which would be a heavy task. We therefore only study the traces of this surface in three orthogonal planes: $\left\{\Sigma_{x x}=\Sigma_{y y} \neq \Sigma_{z z}\right\},\left\{\Sigma_{x x}=\Sigma_{z z} \neq \Sigma_{y y}\right\}$ and $\left\{\Sigma_{y y}=\Sigma_{z z} \neq \Sigma_{x x}\right\}$.

Figure 13 compares the results obtained to those corresponding to the approximate yield locus defined in Section 7. (The quantities plotted on the horizontal and vertical axes are the overall mean and shear stresses normalized by the yield stress). The agreement which can be observed is typical of that obtained in such simulations. It is excellent except near the "hydrostatic point" $\left(\Sigma_{x x}=\Sigma_{y y}=\Sigma_{z z}\right)$; Figures 13(a) and 13(b) show that the numerical yield locus exhibits a slight corner there unlike the approximate one. The presence of this corner is not surprising since the ellipsoidal void considered does not differ much from a circular cylindrical one, for which such a corner has long been known to exist (Pastor and Ponte-Castaneda, 2002).

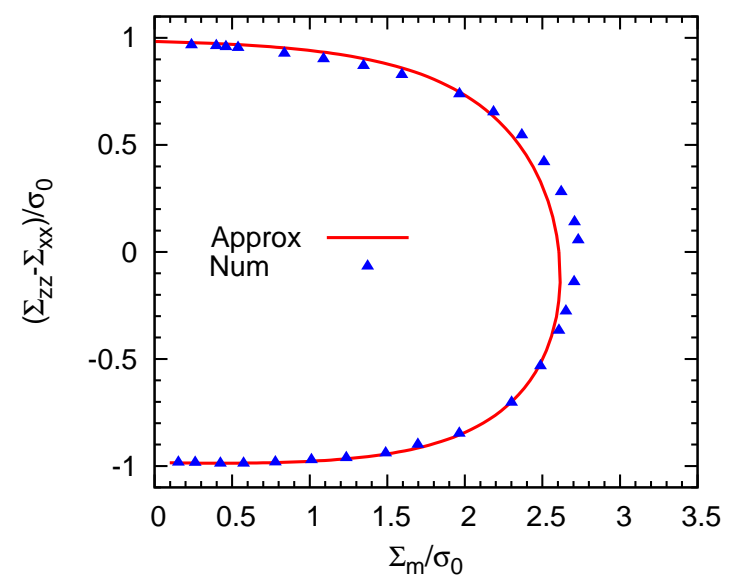

(a) $\Sigma_{x x}=\Sigma_{y y} \neq \Sigma_{z z}, \Sigma_{x y}=\Sigma_{y z}=\Sigma_{z x}=0$

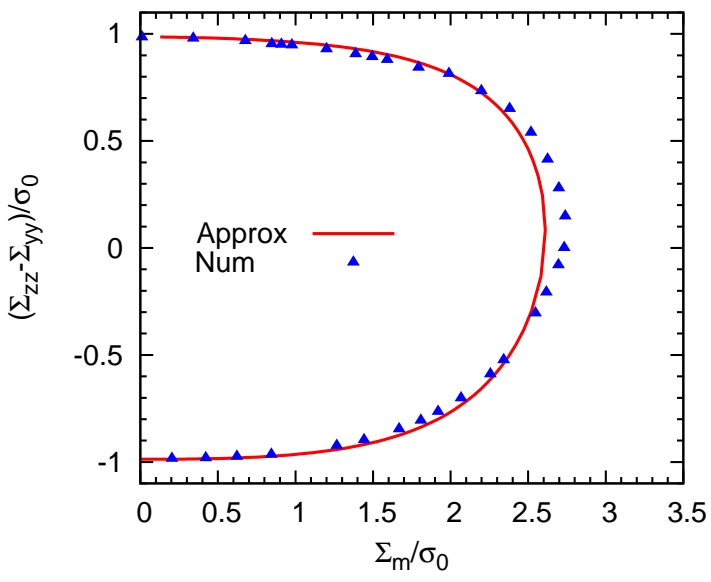

(b) $\Sigma_{x x}=\Sigma_{z z} \neq \Sigma_{y y}, \Sigma_{x y}=\Sigma_{y z}=\Sigma_{z x}=0$

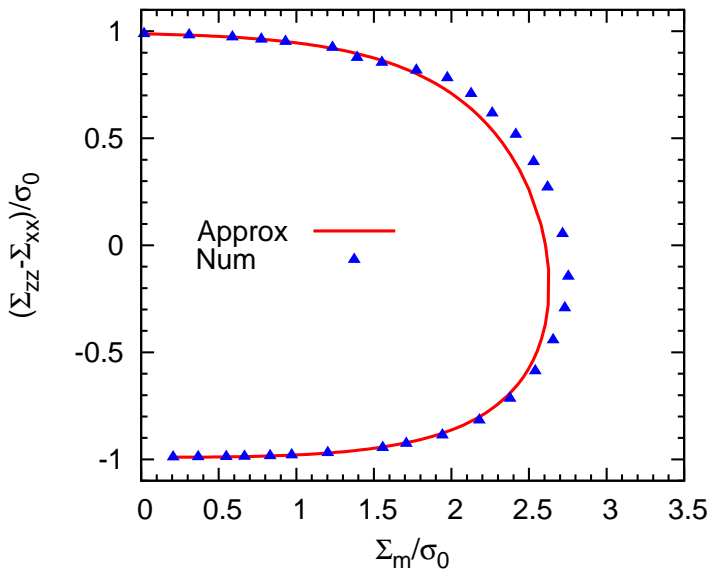

(c) $\Sigma_{y y}=\Sigma_{z z} \neq \Sigma_{x x}, \Sigma_{x y}=\Sigma_{y z}=\Sigma_{z x}=0$

Fig. 13. Traces in three orthogonal planes of the yield locus for a void with semi-axes in the proportions 10:2:1 and a porosity of 0.01 - Numerical results (Num) and approximation proposed (Approx) 


\section{Conclusion}

The work described in this paper represents a second step in the development of a Gursonlike model for porous ductile materials containing arbitrary ellipsoidal voids. Its purpose was to provide explicit expressions of all coefficients of the approximate overall yield criterion developed in Part I. This made it necessary to drop Leblond and Gologanu (2008)'s trial velocity fields satisfying conditions of homogeneous strain rate on confocal ellipsoids, the further use of which would have led to inaccuracies in the case of very flat oblate spheroidal cavities.

Among the coefficients of the yield function, those appearing in the characteristic hyperbolic cosine were determined by using numerical limit-analysis to evaluate, in a number of significant cases, the response of the ellipsoidal cell considered to hydrostatic loadings. Approximate analytical formulae reproducing the results obtained were then defined in order to cover all envisageable cases.

The coefficients appearing in the characteristic quadratic form of the stress components were determined in a more expedient way: the yield function proposed was simply required to coincide, for predominantly deviatoric loadings, with that corresponding to PonteCastaneda (1991)'s, Willis (1991)'s and Michel and Suquet (1992)'s nonlinear extension of Willis (1977)'s bound for voided elastic solids.

The approximate yield criterion being fully defined at this stage, a summary of all its equations was provided for ease of reference.

The model proposed was checked to match a number of exact results or approximate but accurate models pertaining to some significant special cases.

The task undertaken is however still incomplete at this stage. Unavoidable future developments include:

- Assessment of the approximate criterion through numerical calculation of the exact overall yield locus in a number of representative cases. This topic was envisaged here on the basis of a single example, for mere illustrative purposes; a full presentation of comprehensive results obtained in eight different cases will be provided in a future paper.

- Definition of suitable evolution equations for the internal parameters of the model, essentially those characterizing the shape and orientation of the voids. Some results obtained by Aravas and Ponte-Castaneda (2004) using the concept of linear comparison material, completed by numerical simulations, will play a decisive role here.

\section{References}

Aravas N., Ponte Castaneda P. (2004). Numerical methods for porous metals with deformation-induced anisotropy. Comput. Methods Appl. Mech. Engrg., 193, 3767-3805. 
Budiansky B., O'Connell R.J. (1976). Elastic moduli of a cracked solid. Int. J. Solids Structures, 12, 81-97.

de Buhan P., Maghous S. (1995). A straightforward numerical method for evaluating the ultimate loads of structures. Eur. J. Mech. A/Solids, 14, 309-328.

Danas K., Ponte-Castaneda P. (2008a). A finite-strain model for anisotropic viscoplastic porous media: I - Theory. Eur. J. Mech. A/Solids, 28, 387-401.

Eshelby J.D. (1957). The determination of the elastic field of an ellipsoidal inclusion, and related problems. Proc. Roy. Soc. London A, 241, 376-396.

Garajeu M. (1995). Contribution à l'étude du comportement non-linéaire de milieux poreux avec ou sans renfort. Ph.D. Thesis, Université de la Méditerranée, Marseille (in French).

Gologanu M. (1997). Etude de quelques problèmes de rupture ductile des métaux. Ph.D. Thesis, Université Pierre et Marie Curie (Paris VI) (in French).

Gologanu M., Leblond J.B., Devaux J. (1993). Approximate models for ductile metals containing non-spherical voids - Case of axisymmetric prolate ellipsoidal cavities. $J$. Mech. Phys. Solids, 41, 1723-1754.

Gologanu M., Leblond J.B., Devaux J. (1994). Approximate models for ductile metals containing non-spherical voids - Case of axisymmetric oblate ellipsoidal cavities. ASME J. Engng. Materials Technol., 116, 290-297.

Gologanu M., Leblond J.B., Perrin G., Devaux J. (1997). Recent extensions of Gurson's model for porous ductile metals. In: Continuum Micromechanics, P. Suquet, ed., Springer-Verlag, New-York, pp. 61-130.

Gurson A.L. (1977). Continuum theory of ductile rupture by void nucleation and growth: Part I - Yield criteria and flow rules for porous ductile media. ASME J. Engng. Materials Technol., 99, 2-15.

Hill R. (1967). The essential structure of constitutive laws of metal composites and polycristals. J. Mech. Phys. Solids, 15, 79-95.

Leblond J.B., Gologanu M. (2008). External estimate of the yield surface of an arbitrary ellipsoid containing a confocal void. Comptes-Rendus Mécanique, 336, 813-819.

Leblond J.B., Perrin G., Suquet P. (1994). Exact results and approximate models for porous viscoplastic solids. Int. J. Plasticity, 10, 213-235.

Mandel J. (1964). Contribution théorique à l'étude de l'écrouissage et des lois de l'écoulement plastique. In: Proc. 11th Int. Congr. Appl. Mech., Munich, pp. 502-509 (in French).

Michel J.C., Suquet P. (1992). The constitutive law of nonlinear viscous and porous materials. J. Mech. Phys. Solids, 40, 783-812.

Monchiet V., Charkaluk E., Kondo D. (2007). An improvement of Gurson-type models of porous materials by using Eshelby-like trial velocity fields. Comptes-Rendus Mécanique, 335, 32-41.

Monchiet V., Charkaluk E., Kondo D. (2011). A micromechanics-based modification of the Gurson criterion by using Eshelby-like velocity fields. Eur. J. Mech., 30, 940-949.

Monchiet V. (2008). Private communication.

Pastor J. (1978). Analyse-limite : détermination de solutions statiques complètes - Application au talus vertical. J.Méc. Appl., 2, 176-196 (in French).

Pastor F., Loute E., Pastor J., Trillat M. (2009). Mixed method and convex optimization for limit-analysis of homogeneous Gurson materials: a kinematical approach. Eur. J. Mech. A/Solids, 28, 25-35.

Pastor F., Ponte-Castaneda P. (2002). Yield criteria for porous media in plane strain: 
second-order estimates versus numerical results. Comptes-Rendus Mécanique, 330, 741747.

Ponte-Castaneda P. (1991). The effective mechanical properties of nonlinear isotropic materials. J. Mech. Phys. Solids, 39, 45-71.

Ponte-Castaneda P., Suquet P. (1998). Nonlinear composites. Adv. Appl. Mech., 34, 171302.

Ponte-Castaneda P., Willis J. (1995). The effect of spatial distribution on the effective behavior of composite materials and cracked media. J. Mech. Phys. Solids, 43, 19191951.

Ponte-Castaneda P., Zaidman M. (1994). Constitutive models for porous materials with evolving microstructure. J. Mech. Phys. Solids, 42, 1459-1492.

Press W.H., Teukolsky S., Vetterling W., Flannery B. (2007). Numerical Recipes, Third Edition, Cambridge University Press.

Willis J. (1977). Bounds and self-consistent estimates for the overall moduli of anisotropic composites. J. Mech. Phys. Solids, 25, 185-202.

Willis J. (1991). On methods for bounding the overall properties of nonlinear composites. J. Mech. Phys. Solids, 39, 73-86. 


\section{A Appendix: numerical limit-analysis by the finite element method}

The aim of this Appendix is to to study the relation between the equations of limit-analysis and those of the time-discretized finite element problem.

\section{A.1 General case}

The equations of limit-analysis of a structure $\Omega$ are identical to those of a problem of small strain plasticity without elasticity posed on this structure, that is:

$$
\begin{aligned}
& \begin{cases}\operatorname{div} \boldsymbol{\sigma} & =0 \\
\mathbf{d} & =\frac{1}{2}\left[\nabla_{\mathbf{X}} \mathbf{v}+\left(\nabla_{\mathbf{X}} \mathbf{v}\right)^{T}\right] \\
f(\boldsymbol{\sigma}) & \leq 0 \\
\mathbf{d} \quad & =\dot{\eta} \frac{\partial f}{\partial \boldsymbol{\sigma}}(\boldsymbol{\sigma}) \\
\dot{\eta} \quad & \left\{\begin{array}{l}
=0 \text { if } f(\boldsymbol{\sigma})<0 \\
\geq 0 \text { if } f(\boldsymbol{\sigma})=0
\end{array}\right.\end{cases} \\
& + \text { B.C. }
\end{aligned}
$$

In these equations $\mathbf{X}$ denotes the position-vector in the initial configuration, $\mathbf{v}$ the velocity, $\mathbf{d}$ the strain rate, $\boldsymbol{\sigma}$ the Cauchy stress tensor, $f(\boldsymbol{\sigma})$ the von Mises yield function, $\dot{\eta}$ the plastic multiplier, and "B.C." stands for "boundary conditions".

Now assume that the elastoplastic problem is solved by the finite element method using an implicit (backward Euler) algorithm for the projection of the elastic stress predictor onto the yield locus, with a single large step and no geometry update. Let $\boldsymbol{\sigma}^{0}=\mathbf{0}, \boldsymbol{\sigma}^{1}$, $\mathbf{u}^{0}=\mathbf{0}, \mathbf{u}^{1}, \boldsymbol{\epsilon}^{0}=\mathbf{0}, \boldsymbol{\epsilon}^{1}$ denote the initial and final stresses, initial and final displacements, initial and final strains, respectively. Provided that the load increment is large enough for the order of magnitude of the elastic strains in the structure to be much smaller than that of the plastic strains, the equations of the time-discretized problem read

$$
\begin{aligned}
& \begin{cases}\operatorname{div} \boldsymbol{\sigma}^{1} & =0 \\
\boldsymbol{\epsilon}^{1} & =\frac{1}{2}\left\{\nabla_{\mathbf{X}}\left(\mathbf{u}^{1}\right)+\left[\nabla_{\mathbf{X}}\left(\mathbf{u}^{1}\right)\right]^{T}\right\} \\
f\left(\boldsymbol{\sigma}^{1}\right) & \leq 0 \\
\boldsymbol{\epsilon}^{1}-\boldsymbol{\epsilon}^{0}=\boldsymbol{\epsilon}^{1} & \simeq \Delta \eta \frac{\partial f}{\partial \boldsymbol{\sigma}}\left(\boldsymbol{\sigma}^{1}\right) \\
\Delta \eta & \left\{\begin{array}{l}
=0 \text { if } f\left(\boldsymbol{\sigma}^{1}\right)<0 \\
\geq 0
\end{array}\right.\end{cases} \\
& \text { +B.C. }
\end{aligned}
$$


where $\Delta \eta$ denotes the discretized plastic multiplier. The equivalence of systems (A.1) and (A.2) is clear ${ }^{12}$, with the correspondences $\boldsymbol{\sigma} \leftrightarrow \boldsymbol{\sigma}^{1}, \mathbf{v} \leftrightarrow \mathbf{u}^{1}, \mathbf{d} \leftrightarrow \boldsymbol{\epsilon}^{1}$.

Thus one may solve a problem of limit-analysis by the standard finite element method, with a single load step sufficiently large to ensure that the elastic strains in the structure are generally much smaller than the plastic strains. From the finite element results may be deduced not only the limit-load (from the stresses), but also the velocity field (proportional to the displacement field) and the strain rate field (proportional to the strain field).

\section{A.2 Special case of a completely plastic structure}

We now consider the special but frequent case where the structure is entirely plastic when its limit-load is reached. Equations (A.1) $)_{3,4,5}$ of the limit-analysis problem may then be simplified into

$$
\left\{\begin{array}{l}
f(\boldsymbol{\sigma})=0 \\
\mathbf{d} \quad=\dot{\eta} \frac{\partial f}{\partial \boldsymbol{\sigma}}(\boldsymbol{\sigma})=\frac{3}{2} \frac{\dot{\eta}}{\sigma_{e q}} \boldsymbol{\sigma}^{\prime}, \quad \dot{\eta}>0 \quad \text { in } \Omega
\end{array}\right.
$$

where account has been taken of the expression of von Mises's yield function, $f(\boldsymbol{\sigma}) \equiv$ $\sigma_{e q}-\sigma_{0}$ where $\sigma_{e q}$ is the equivalent stress and $\sigma_{0}$ the yield stress in simple tension, and $\boldsymbol{\sigma}^{\prime}$ denotes the deviator of $\boldsymbol{\sigma}$.

Assume again that the elastoplastic problem is solved by the finite element method, but now using a value of Poisson's ratio very close to $1 / 2$ and a single step sufficiently large to ensure that the limit-load is reached, but not necessarily that the final elastic strain $\boldsymbol{\epsilon}^{e 1}$ is much smaller than the final plastic strain $\boldsymbol{\epsilon}^{p 1}$. Under such conditions $\operatorname{tr} \boldsymbol{\epsilon}^{e 1} \simeq 0$ and therefore $\boldsymbol{\epsilon}^{e 1} \simeq\left(\boldsymbol{\sigma}^{1}\right)^{\prime} /(2 \mu)$ where $\left(\boldsymbol{\sigma}^{1}\right)^{\prime}$ is the deviator of $\boldsymbol{\sigma}^{1}$ and $\mu$ the elastic shear modulus; equations (A.2) $)_{3,4,5}$ of the discretized problem then become

$$
\left\{\begin{array}{l}
f\left(\boldsymbol{\sigma}^{1}\right)=0 \\
\boldsymbol{\epsilon}^{1}-\boldsymbol{\epsilon}^{0}=\boldsymbol{\epsilon}^{1}=\boldsymbol{\epsilon}^{e 1}+\boldsymbol{\epsilon}^{p 1}=\frac{\left(\boldsymbol{\sigma}^{1}\right)^{\prime}}{2 \mu}+\Delta \eta \frac{\partial f}{\partial \boldsymbol{\sigma}}\left(\boldsymbol{\sigma}^{1}\right)=\left[\frac{1}{2 \mu}+\frac{3}{2} \frac{\Delta \eta}{\sigma_{e q}}\right]\left(\boldsymbol{\sigma}^{1}\right)^{\prime}, \Delta \eta>0
\end{array} \text { in } \Omega .\right.
$$

The term [...] here is positive since both $\mu$ and $\Delta \eta$ are positive. It follows that a solution of the finite element equations (A.2) ${ }_{1,2,6}-(\text { A.4) is also a solution of the equations (A.1) })_{1,2,6}$ - (A.3) of the theoretical problem, with the same correspondences $\boldsymbol{\sigma} \leftrightarrow \boldsymbol{\sigma}^{1}, \mathbf{v} \leftrightarrow \mathbf{u}^{1}$, $\mathbf{d} \leftrightarrow \boldsymbol{\epsilon}^{1}$ as before.

Thus, if the structure is entirely plastic when its limit-load is reached, a finite element solution of the problem of limit-analysis may be obtained by using a value of Poisson's ratio close to $1 / 2$ and a single, moderately large loading step ensuring that the limit-load

\footnotetext{
$\overline{12}$ The keypoint here is that $\frac{\partial f}{\partial \boldsymbol{\sigma}}$ is taken at the point $\boldsymbol{\sigma}^{1}$ rather than $\boldsymbol{\sigma}^{0}=\mathbf{0}$ in the discretized flow rule.
} 
is reached but not that the elastic strain is negligible. ${ }^{13}$ It is however important to note that if such a procedure is used, the strain-rate of limit-analysis corresponds to the total strain of the finite element calculation, not the plastic strain as one would intuitively expect.

\section{B Appendix: the nonlinear Willis bound for plastic voided solids}

To express the Willis bound in the simplest possible format, one must distinguish between the diagonal and off-diagonal components of the overall stress tensor $\boldsymbol{\Sigma}$ by defining the vectors

$$
\Sigma^{\mathrm{dg}} \equiv\left(\begin{array}{c}
\Sigma_{x x} \\
\Sigma_{y y} \\
\Sigma_{z z}
\end{array}\right) \quad ; \quad \Sigma^{\mathrm{offdg}} \equiv\left(\begin{array}{c}
\Sigma_{x y} \\
\Sigma_{y z} \\
\Sigma_{z x}
\end{array}\right) .
$$

For an ideal-plastic material, Willis's quadratic form $\mathcal{Q}^{W}(\boldsymbol{\Sigma})$ defining his approximate yield criterion in the form (31) then reads

$$
\mathcal{Q}^{W}(\boldsymbol{\Sigma}) \equiv \boldsymbol{\Sigma}^{\mathrm{dg}} \cdot \mathbf{M}^{\mathrm{dg}} \cdot \boldsymbol{\Sigma}^{\mathrm{dg}}+\boldsymbol{\Sigma}^{\mathrm{offdg}} \cdot \mathbf{M}^{\mathrm{offdg}} \cdot \boldsymbol{\Sigma}^{\mathrm{offdg}}
$$

where $\mathbf{M}^{\mathrm{dg}}$ and $\mathbf{M}^{\text {offdg }}$ are symmetric second-rank tensors defined by

$$
\begin{gathered}
\mathbf{M}^{\mathrm{dg}} \equiv(1-f)\left[\begin{array}{rrr}
1 & -\frac{1}{2} & -\frac{1}{2} \\
-\frac{1}{2} & 1 & -\frac{1}{2} \\
-\frac{1}{2} & -\frac{1}{2} & 1
\end{array}\right]+\frac{3 f}{2}\left[\begin{array}{ccc}
T_{x x x x} & T_{x x y y} & T_{x x z z} \\
T_{y y x x} & T_{y y y y} & T_{y y z z} \\
T_{z z x x} & T_{z z y y} & T_{z z z z}
\end{array}\right]^{-1} ; \\
\mathbf{M}^{\mathrm{offdg}} \equiv 3(1-f)\left[\begin{array}{lll}
1 & 0 & 0 \\
0 & 1 & 0 \\
0 & 0 & 1
\end{array}\right]+\frac{3 f}{2}\left[\begin{array}{ccc}
T_{x y x y} & 0 & 0 \\
0 & T_{y z y z} & 0 \\
0 & 0 & T_{z x z x}
\end{array}\right]^{-1} .
\end{gathered}
$$

In equations (B.3) and (B.4), $\mathbf{T}$ is a fourth-rank tensor connected to the Eshelby tensor $\mathbf{S}(\nu)$ of an ellipsoidal void of semi-axes $a, b, c$ embedded in a fictitious infinite elastic medium with shear modulus $\mu$ and Poisson ratio $\nu$, through the relation

$$
\mathbf{T} \equiv \lim _{\nu \rightarrow 1 / 2} \frac{1}{2 \mu} \mathbf{L}(\mu, \nu):[\mathbf{I}-\mathbf{S}(\nu)]
$$

where $\mathbf{L}(\mu, \nu)$ denotes the stiffness tensor of the medium and $\mathbf{I}$ the identity tensor. The tensor $\mathbf{T}$ is very closely connected to Aravas and Ponte-Castaneda (2004)'s tensor $\mathbf{Q} \equiv$ $\mathbf{L}(\mu, \nu):[\mathbf{I}-\mathbf{S}(\nu)]$; it is more relevant in the present context since it is independent of the

\footnotetext{
$\overline{13}$ The reason why this does not remain true if the structure is only partially plastic is that the term [...] of equation (A.4) $)_{2}$ is then positive everywhere whereas the plastic multiplier $\dot{\eta}$ of equation (A.3) $)_{2}$ is so only at those points where $f(\boldsymbol{\sigma})=0$.
} 
fictitious shear modulus $\mu$. Its components obey the symmetry relations $T_{i j k l}=T_{j i k l}=$ $T_{i j l k}=T_{k l i j}$ and are listed in Appendix $\mathrm{C}$ below.

\section{Appendix: components of the tensor $\mathrm{T}$}

The components of the tensor $\mathbf{T}$ defined by equation (B.5) are easily obtained from those of the tensor $\mathbf{Q}$ provided by Aravas and Ponte-Castaneda (2004). They are given by

$$
\left\{\begin{array}{l}
T_{x x x x} \equiv 2-\frac{3 a^{2} I_{a a}+I_{a}}{4 \pi} \\
T_{x x y y} \equiv 1-\frac{I_{a}+I_{b}+3\left(a^{2}+b^{2}\right) I_{a b}}{8 \pi} \\
T_{x y x y} \equiv \frac{1}{2}-\frac{3\left(a^{2}+b^{2}\right) I_{a b}}{8 \pi},
\end{array}\right.
$$

plus similar formulae resulting from simultaneous cyclic interchange of $x, y, z$ and $a, b, c$. In these expressions $I_{a}, I_{a a}, I_{a b}$, etc. are Eshelby (1957)'s integrals defined by

$$
\left\{\begin{aligned}
I_{a} & \equiv 2 \pi a b c \int_{0}^{+\infty} \frac{d \rho}{\left(a^{2}+\rho\right) v(\rho)} \\
I_{a a} & \equiv 2 \pi a b c \int_{0}^{+\infty} \frac{d \rho}{\left(a^{2}+\rho\right)^{2} v(\rho)} \\
I_{a b} & \equiv \frac{2 \pi}{3} a b c \int_{0}^{+\infty} \frac{d \rho}{\left(a^{2}+\rho\right)\left(b^{2}+\rho\right) v(\rho)}
\end{aligned}\right.
$$

and other similar formulae, where $v(\rho)$ denotes the function defined by equation (4) of Part I.

As explained by Eshelby (1957), the relations

$$
\begin{cases}I_{a}+I_{b}+I_{c} & =4 \pi \\ I_{a a}+I_{a b}+I_{a c} & =\frac{4 \pi}{3 a^{2}} \\ I_{a b} & =-\frac{I_{a}-I_{b}}{3\left(a^{2}-b^{2}\right)}\end{cases}
$$

plus similar ones, permit to deduce all integrals from only two among $I_{a}, I_{b}$ and $I_{c}$. The procedure is as follows.

- First, the third integral of type $I_{a}$ may be deduced from the other two through equation (C.3) ${ }_{1}$.

- The integrals $I_{a b}, I_{a c}, I_{b c}$ may next be calculated. If $a>b>c$, it suffices to do so to use equation (C.3 $)_{3}$ and similar ones. If $a=b>c$, equation (C.3) ${ }_{3}$ for $I_{a b}$ breaks down, but its equivalents for $I_{a c}$ and $I_{b c}$ don't, and $I_{a b}$ may be deduced from the obvious relation $I_{a a}=3 I_{a b}$ combined with equation (C.3) $)_{2}$. If $a>b=c$, one may similarly calculate $I_{a b}$ and $I_{a c}$ directly, and $I_{b c}$ from the equality $I_{b b}=3 I_{b c}$ combined with an equation similar to (C.3) $)_{2}$. Finally if $a=b=c$, equation (C.3) $)_{3}$ breaks down for all integrals, 
but equation (C.3 $)_{2}$ and similar ones, combined with obvious equalities, imply that $I_{a b}=I_{a c}=I_{b c}=4 \pi /\left(15 a^{2}\right)$.

- In a third step the integrals $I_{a a}, I_{b b}, I_{c c}$ follow from equation (C.3) $)_{2}$ and similar ones.

To calculate two of the integrals $I_{a}, I_{b}, I_{c}$, the most economical method probably consists in relating them to Carlson's function

$$
R_{D}(x, y, z) \equiv \frac{3}{2} \int_{0}^{+\infty} \frac{d t}{\sqrt{(x+t)(y+t)(z+t)^{3}}}
$$

through the relation

$$
I_{a}=\frac{4 \pi}{3} a b c R_{D}\left(b^{2}, c^{2}, a^{2}\right)
$$

plus similar ones. Short and extremely efficient routines in FORTRAN, C and $\mathrm{C}^{++}$may be found in Press et al. (2007)'s book to calculate the function $R_{D}$. 